\title{
Angular correlations in the double Drell-Yan process
}

\author{
Tomas Kasemets and Markus Diehl \\ Deutsches Elektronen-Synchroton DESY, \\ 22603 Hamburg, Germany \\ E-mail: tomas.kasemets@desy.de, markus.diehl@desy.de
}

\begin{abstract}
We study the impact of parton correlations on the double Drell-Yan process, i.e. on the production of two electroweak gauge bosons by double parton scattering in a single proton-proton collision. Spin correlations between two partons in a proton are shown to change the overall rate of the process and to induce characteristic angular correlations between the decay leptons of the two gauge bosons.
\end{abstract}

Keywords: QCD Phenomenology, Hadronic Colliders

ARXIV EPRINT: 1210.5434 


\section{Contents}

1 Introduction 1

2 Double parton scattering $\quad 2$

2.1 Double parton distributions 4

$\begin{array}{ll}2.2 \text { Reference frames } & 6\end{array}$

2.3 Hard-scattering cross sections 8

3 The double Drell-Yan cross section $\quad 10$

$\begin{array}{ll}\text { 3.1 Cross section integrated over transverse boson momenta } & 15\end{array}$

4 Transverse position dependence of distributions $\quad 16$

$\begin{array}{llr}5 & \text { Summary } & 18\end{array}$

$\begin{array}{ll}\text { A Coupling factors } & \mathbf{1 9}\end{array}$

$\begin{array}{ll}\text { A.1 Charged vector bosons } & 19\end{array}$

$\begin{array}{lll}\text { A.2 Neutral vector bosons } & 20\end{array}$

\section{Introduction}

In the era of the LHC, a thorough understanding of the strong-interaction dynamics in proton-proton collisions is an imperative. Whereas the determination of parton distribution functions and the computation of hard-scattering processes at parton level are becoming fields of precision physics, other aspects remain much less well understood, both at a conceptual and a practical level. Among these aspects are multiparton interactions, where in a single proton-proton collision more than one parton in each proton participates in a hard scattering. Multiparton interactions can give sizeable contributions both to signal and to background processes, notably to Higgs production [1], to electroweak processes [2-10] and to multijet production [11-18]. They are also of interest in their own right from the point of view of hadron structure, because they contain information about correlations between partons inside the proton.

Experimental evidence for hard multiparton interactions has been found at the ISR [19], the SPS [20] and the Tevatron [21-25]. Due to the rapid increase of parton densities at small momentum fractions, one expects these interactions to be even more prominent at the LHC, and first results have indeed been reported [26], with more studies being expected in the future [27]. Substantial effort has gone into the modeling and implementation of multiparton interactions in Monte-Carlo generators [28-34]. A mini-review on earlier developments of the subject is given in [31], and an overview of current developments can be found in the conference proceedings $[35,36]$. Recently there has been renewed interest in 
understanding the theoretical foundations of multiple hard scattering [37-47], with many issues remaining to be clarified or worked out.

The simplest assumption one can make when computing multiple hard scattering is that the different partons in the proton are uncorrelated with each other. This leads to very compact results, but it certainly is a simplification whose validity and limitations need to be investigated. Recent phenomenological studies of different types of correlation effects in multiparton interactions can be found in [17, 34, 48-52]. In this work we focus on correlations between the polarization of two partons in an unpolarized proton and on their consequences for the overall rate and for final-state distributions in double hard scattering processes. We build on the observations made in [40] and extend the results of [46]. The relevance of spin correlations for multiple interactions was pointed out long ago in [53], but that work have not been followed up until recently.

We perform our study for double Drell-Yan production, where two electroweak gauge bosons $\left(\gamma^{*}, Z, W^{ \pm}\right)$are produced in two independent quark-antiquark annihilation processes. This has long been recognized as a prototype for multi-parton interactions [54-56]. Unfortunately, recent phenomenological estimates $[8,9,57]$ find that rates for double DrellYan production at the LHC will likely be too small to allow for a detailed experimental investigation of final-state distributions. We nevertheless choose this process for our investigation, given that it is among the simplest double scattering process from the theoretical point of view but still exhibits a wealth of nontrivial features. The results we obtain for double Drell-Yan production will have their analogs for processes with higher rates, such as the production of four jets or the production of one gauge boson plus a dijet. (Note that up to a global factor the tree-level graph for the production and subsequent decay of a gauge boson is identical to the graph for $q_{1} \bar{q}_{2} \rightarrow q_{3} \bar{q}_{4}$ with a gluon in the $s$-channel.)

Even if two partons in a proton are unpolarized, there can be correlations between their transverse positions and their longitudinal momentum fractions. There are indeed reasons to expect such correlations, see e.g. [34] and section 2.6 of [40], but not much is currently known about them. In the spirit of an exploratory study, we will calculate these correlations in a simple model of the proton and discuss their consequence for the transverse-momentum spectrum of double Drell-Yan production.

This paper is organized as follows. In the next section we recall the basic formalism for computing double parton interactions, define double parton distributions that describe spin correlations between the two partons and give the polarization dependent parton-level cross sections. In section 3 we give our results for the cross section of the double Drell-Yan process. In section 4 we investigate the correlation between transverse and longitudinal variables in double parton distributions within a simple model. Section 5 summarizes our findings, and in an appendix we list the coupling factors entering the cross section formulae in section 3 .

\section{Double parton scattering}

Consider the production of two gauge bosons $V_{1}, V_{2}=\gamma^{*}, Z, W$ in a $p p$ collision, followed by the leptonic decays $\gamma^{*}, Z \rightarrow \ell^{+} \ell^{-}$or $W \rightarrow \ell \nu$. Four-momenta are assigned as $p(p)+$ 
$p(\bar{p}) \rightarrow V_{1}\left(q_{1}\right)+V_{2}\left(q_{2}\right)+X$. We are interested in the fully differential cross section of the four-lepton final state and restrict ourselves to the kinematic region where the transverse momenta $\boldsymbol{q}_{1}$ and $\boldsymbol{q}_{2}$ of the gauge bosons in the $p p$ center-of-mass are much smaller than their invariant masses, i.e. we assume $\boldsymbol{q}_{1}^{2}, \boldsymbol{q}_{2}^{2} \ll q_{1}^{2}, q_{2}^{2}$. It is in this kinematics that double parton scattering is not power suppressed compared with the production of the gauge boson pair by a single hard scattering [39]. In the calculation of the cross section, the invariant masses $Q_{i}=\left(q_{i}^{2}\right)^{1 / 2}$ will serve as the hard scale necessary for the application of factorization. For simplicity we shall not assume any particular hierarchy in size between $\boldsymbol{q}_{1}$ and $\boldsymbol{q}_{2}$ or between $Q_{1}$ and $Q_{2}$.

We assume that the double hard scattering cross section factorizes into the product of a double parton distribution (DPD) in each proton and a parton-level cross section for each of the two hard scatters. This factorization has not been proven, but several elements of such a proof have been given in [40]. Schematically, the double parton scattering cross section then reads $[39,40]$

$$
\begin{aligned}
\left.\frac{d \sigma}{\prod_{i=1}^{2} d x_{i} d \bar{x}_{i} d^{2} \boldsymbol{q}_{i} d \Omega_{i}}\right|_{\mathrm{DPS}}= & \frac{1}{C} \frac{d \hat{\sigma}_{1}}{d \Omega_{1}} \frac{d \hat{\sigma}_{2}}{d \Omega_{2}} \int \frac{d^{2} \boldsymbol{z}_{1}}{(2 \pi)^{2}} \frac{d^{2} \boldsymbol{z}_{2}}{(2 \pi)^{2}} e^{-i \boldsymbol{z}_{1} \boldsymbol{q}_{1}-i \boldsymbol{z}_{2} \boldsymbol{q}_{2}} \\
& \times \int d^{2} \boldsymbol{y} F\left(x_{1}, x_{2}, \boldsymbol{z}_{1}, \boldsymbol{z}_{2}, \boldsymbol{y}\right) \bar{F}\left(\bar{x}_{1}, \bar{x}_{2}, \boldsymbol{z}_{1}, \boldsymbol{z}_{2}, \boldsymbol{y}\right)
\end{aligned}
$$

with a combinatorial factor $C$ equal to 2 when the final states of the two hard interactions are identical and equal to 1 otherwise. Here $d \hat{\sigma}_{i} / d \Omega_{i}$ denotes the cross section for quarkantiquark annihilation into a lepton pair via the gauge boson $V_{i}$, taken differential w.r.t. the lepton angles in the appropriate boson rest frame (see section 2.2). In the $p p$ centerof-mass we define the $z$ axis to point into the direction of the proton momentum $p$ and use light-cone coordinates $v^{ \pm}=\left(v^{0} \pm v^{3}\right) / \sqrt{2}$ and $\boldsymbol{v}=\left(v^{1}, v^{2}\right)$ for any four-vector $v$. The kinematic variables $x_{i}=q_{i}^{+} / p^{+}$and $\bar{x}_{i}=q_{i}^{-} / \bar{p}^{-}$determine the longitudinal parton momentum fractions in the DPDs, which we denote by $F$ for the proton with momentum $p$ and by $\bar{F}$ for the proton with momentum $\bar{p}$. The arguments $\boldsymbol{z}_{i}$ and $\boldsymbol{y}$ of the distributions determine where the hard-scattering processes take place in transverse configuration space. As indicated in figure $1, \boldsymbol{y}$ is the transverse distance between the two scattering partons in a proton (and hence between the two annihilation processes) if one takes the average between the scattering amplitude and its conjugate. As shown in $[39,40] \boldsymbol{z}_{i}$ is the Fourier conjugate variable to the transverse momentum of parton $i$ (again averaged between amplitude and conjugate amplitude). $F\left(x_{1}, x_{2}, \boldsymbol{z}_{1}, \boldsymbol{z}_{2}, \boldsymbol{y}\right)$ is thus the Fourier transform of a transversemomentum dependent DPD. The factorization formula (2.1) generalizes the expression for single Drell-Yan production in terms of transverse-momentum dependent single-parton densities $[58,59]$.

Integrating (2.1) over the transverse boson momenta $\boldsymbol{q}_{1}$ and $\boldsymbol{q}_{2}$ one obtains

$$
\left.\frac{d \sigma}{\prod_{i=1}^{2} d x_{i} d \bar{x}_{i} d \Omega_{i}}\right|_{\mathrm{DPS}}=\frac{1}{C} \frac{d \hat{\sigma}_{1}}{d \Omega_{1}} \frac{d \hat{\sigma}_{2}}{d \Omega_{2}} \int d^{2} \boldsymbol{y} F\left(x_{1}, x_{2}, \boldsymbol{y}\right) \bar{F}\left(\bar{x}_{1}, \bar{x}_{2}, \boldsymbol{y}\right) .
$$

Here $F\left(x_{1}, x_{2}, \boldsymbol{y}\right)$ and $\bar{F}\left(\bar{x}_{1}, \bar{x}_{2}, \boldsymbol{y}\right)$ are transverse-momentum integrated (also called collinear) DPDs, which were introduced long ago in [55,60]. Naively, they are obtained by 


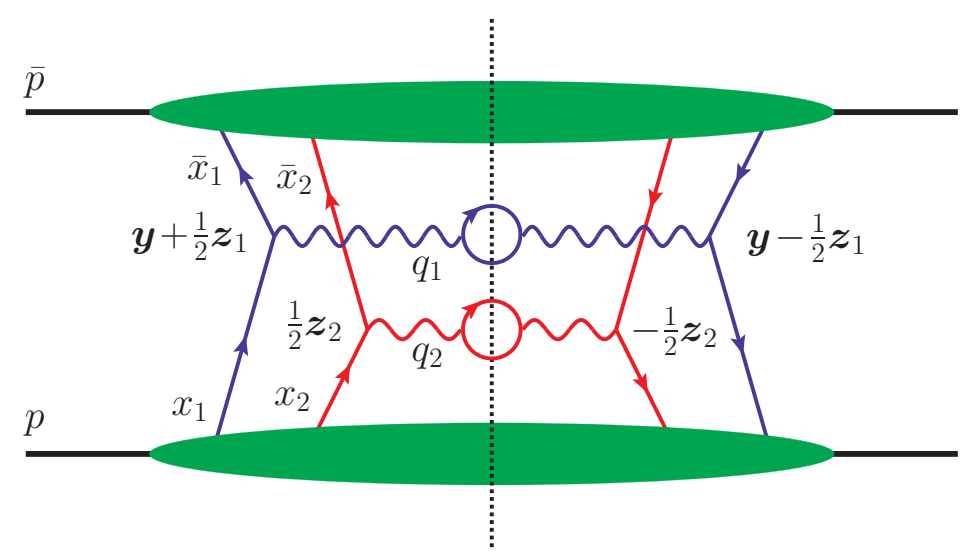

Figure 1. A graph for the double Drell-Yan process, where two quarks in the right-moving proton interact with two antiquarks in the left-moving proton. The figure shows the assignment of four-momenta $\left(p, \bar{p}, q_{1}, q_{2}\right)$, of light-cone momentum fractions $\left(x_{i}, \bar{x}_{i}\right)$ and of transverse position arguments as explained in the text. The dotted vertical line denotes the final-state cut.

setting $\boldsymbol{z}_{1}=\boldsymbol{z}_{2}=\mathbf{0}$ in the distributions that appear in (2.1). Closer analysis reveals that transverse-momentum dependent and collinear DPDs (just as their counterparts for single partons) require different types of regularization and subtractions of divergences. As a result the distributions depend in different ways on an ultraviolet renormalization scale, and (with the exception of specific distributions) also on a rapidity parameter which is closely related to Sudakov logarithms. This is discussed in [40] and will be tacitly implied in the remainder of the present work.

Equations (2.1) and (2.2) are schematic in that they omit labels for and summation over the quantum numbers of the partons (quarks vs. antiquarks, flavor, polarization and color). This information will be restored in section 2.3. We emphasize that these equations only give one contribution to the cross section for four-lepton production. Further contributions need to be added from the familiar single hard-scattering mechanism (where the four leptons are produced in a single parton-level process), the interference between single and double hard scattering, as well as double hard-scattering graphs with fermion number interference [40]. The single hard-scattering contribution is straightforward to compute (see e.g. [57]), whereas the different interference contributions are not. As argued in [40], the fermion number interference contribution should become relatively unimportant at small momentum fractions $x_{i}, \bar{x}_{i}$.

\subsection{Double parton distributions}

We now briefly specify the DPDs necessary for our study, referring to [40] for more detail. For two quarks in an unpolarized right-moving proton we write

$$
F_{a_{1} a_{2}}\left(x_{1}, x_{2}, \boldsymbol{z}_{1}, \boldsymbol{z}_{2}, \boldsymbol{y}\right)=2 p^{+} \int \frac{d z_{1}^{-}}{2 \pi} \frac{d z_{2}^{-}}{2 \pi} d y^{-} e^{i x_{1} z_{1}^{-} p^{+}+i x_{2} z_{2}^{-} p^{+}}\left\langle p\left|\mathcal{O}_{a_{2}}\left(0, z_{2}\right) \mathcal{O}_{a_{1}}\left(y, z_{1}\right)\right| p\right\rangle
$$


where an average over the proton polarization is implied. The operators

$$
\mathcal{O}_{a_{i}}\left(y, z_{i}\right)=\left.\bar{q}_{i}\left(y-\frac{1}{2} z_{i}\right) \Gamma_{a_{i}} q_{i}\left(y+\frac{1}{2} z_{i}\right)\right|_{z_{i}^{+}=y^{+}=0}
$$

for quarks of flavor $q_{i}$ are understood to include appropriate Wilson lines between the two quark fields, as well as appropriate regularization and subtractions as mentioned above. Notice the correspondence of position arguments in (2.3) and (2.4) with figure 1. The Dirac matrices

$$
\Gamma_{q}=\frac{1}{2} \gamma^{+}, \quad \Gamma_{\Delta q}=\frac{1}{2} \gamma^{+} \gamma_{5}, \quad \Gamma_{\delta q}^{j}=\frac{1}{2} i \sigma^{j+} \gamma_{5} \quad(j=1,2)
$$

select different polarizations of the quarks in the proton, with $q$ corresponding to unpolarized quarks, $\Delta q$ corresponding to longitudinal polarization and $\delta q$ to polarization in the transverse direction $j$. Notice that the labels $a_{i}$ in (2.3) specify both the flavor and the polarization of the quarks. In full analogy one can define DPDs $F_{\bar{a}_{1}, \bar{a}_{2}}$ for two antiquarks, as well as quark-antiquark distributions $F_{\bar{a}_{1}, a_{2}}$ and $F_{a_{1}, \bar{a}_{2}}$.

The quark coupling to gauge boson $V_{1}$ need not have the same flavor in the scattering amplitude and its complex conjugate, because a mismatch in flavor can be compensated by the quark coupling to gauge boson $V_{2}$. For example, the quarks with transverse positions $\boldsymbol{y}+\frac{1}{2} \boldsymbol{z}_{1}$ and $-\frac{1}{2} \boldsymbol{z}_{2}$ in figure 1 can be $u$ quarks if the quarks with transverse positions $\frac{1}{2} \boldsymbol{z}_{2}$ and $\boldsymbol{y}-\frac{1}{2} \boldsymbol{z}_{1}$ are $d$ quarks. The DPDs describing this type of quark flavor interference are given by

$$
F_{a_{1} a_{2}}^{I}\left(x_{1}, x_{2}, \boldsymbol{z}_{1}, \boldsymbol{z}_{2}, \boldsymbol{y}\right)=2 p^{+} \int \frac{d z_{1}^{-}}{2 \pi} \frac{d z_{2}^{-}}{2 \pi} d y^{-} e^{i x_{1} z_{1}^{-} p^{+}+i x_{2} z_{2}^{-} p^{+}}\left\langle p\left|\mathcal{O}_{a_{2}}^{I}\left(0, z_{2}\right) \mathcal{O}_{a_{1}}^{I}\left(y, z_{1}\right)\right| p\right\rangle
$$

with the product of operators

$$
\mathcal{O}_{a_{2}}^{I}\left(0, z_{2}\right) \mathcal{O}_{a_{1}}^{I}\left(y, z_{1}\right)=\left.\bar{q}_{1}\left(-\frac{1}{2} z_{2}\right) \Gamma_{a_{2}} q_{2}\left(\frac{1}{2} z_{2}\right) \bar{q}_{2}\left(y-\frac{1}{2} z_{1}\right) \Gamma_{a_{1}} q_{1}\left(y+\frac{1}{2} z_{1}\right)\right|_{z_{1}^{+}=z_{2}^{+}=y^{+}=0} .
$$

We note that these distributions are complex valued and that their imaginary part changes sign when one interchanges the flavor (but not the spin) assignments and replaces $\boldsymbol{z}_{i} \rightarrow-\boldsymbol{z}_{i}$, e.g.

$$
F_{q_{1} \Delta q_{2}}^{I}\left(x_{1}, x_{2}, \boldsymbol{z}_{1}, \boldsymbol{z}_{2}, \boldsymbol{y}\right)=\left[F_{q_{2} \Delta q_{1}}^{I}\left(x_{1}, x_{2},-\boldsymbol{z}_{1},-\boldsymbol{z}_{2}, \boldsymbol{y}\right)\right]^{*} .
$$

As we shall see, this ensures that physical cross sections are real-valued.

Let us now classify the different combinations of quark polarization, taking into account the constraints of parity invariance [40]. For unpolarized and longitudinally polarized quarks we have

$$
\begin{aligned}
F_{q q} & =f_{q q}\left(x_{1}, x_{2}, \boldsymbol{z}_{1}, \boldsymbol{z}_{2}, \boldsymbol{y}\right), & F_{\Delta q \Delta q} & =f_{\Delta q \Delta q}\left(x_{1}, x_{2}, \boldsymbol{z}_{1}, \boldsymbol{z}_{2}, \boldsymbol{y}\right), \\
F_{q \Delta q} & =g_{q \Delta q}\left(x_{1}, x_{2}, \boldsymbol{z}_{1}, \boldsymbol{z}_{2}, \boldsymbol{y}\right), & F_{\Delta q q} & =g_{\Delta q q}\left(x_{1}, x_{2}, \boldsymbol{z}_{1}, \boldsymbol{z}_{2}, \boldsymbol{y}\right),
\end{aligned}
$$


where $f$ denotes scalar and $g$ pseudoscalar functions. For transverse quark polarization the parton distributions carry a transverse index and can be decomposed as

$$
\begin{aligned}
F_{\Delta q \delta q}^{i} & =M\left(\boldsymbol{y}^{i} f_{\Delta q \delta q}+\tilde{\boldsymbol{y}}^{i} g_{\Delta q \delta q}\right), & F_{\delta q \Delta q}^{i} & =M\left(\boldsymbol{y}^{i} f_{\delta q \Delta q}+\tilde{\boldsymbol{y}}^{i} g_{\delta q \Delta q}\right), \\
F_{q \delta q}^{i} & =M\left(\tilde{\boldsymbol{y}}^{i} f_{q \delta q}+\boldsymbol{y}^{i} g_{q \delta q}\right), & F_{\delta q q}^{i} & =M\left(\tilde{\boldsymbol{y}}^{i} f_{\delta q q}+\boldsymbol{y}^{i} g_{\delta q q}\right),
\end{aligned}
$$

where the scalar and pseudoscalar functions depend on the same variables as in (2.9). Here $\tilde{\boldsymbol{y}}^{i}=\epsilon^{i j} \boldsymbol{y}^{j}$ is a transverse vector orthogonal to $\boldsymbol{y}^{i}$, defined in terms of the two-dimensional antisymmetric tensor $\epsilon^{i j}$ (with $\epsilon^{12}=1$ ). Factors of the proton mass $M$ have been introduced in order to have the same mass dimension for all distributions $f$ and $g$. For two transversely polarized quarks we finally write

$$
\begin{aligned}
F_{\delta q \delta q}^{i j}= & \delta^{i j} f_{\delta q \delta q}+M^{2}\left(2 \boldsymbol{y}^{i} \boldsymbol{y}^{j}-\boldsymbol{y}^{2} \delta^{i j}\right) f_{\delta q \delta q}^{t} \\
& +M^{2}\left(\boldsymbol{y}^{i} \tilde{\boldsymbol{y}}^{j}+\tilde{\boldsymbol{y}}^{i} \boldsymbol{y}^{j}\right) g_{\delta q \delta q}^{s}+M^{2}\left(\boldsymbol{y}^{i} \tilde{\boldsymbol{y}}^{j}-\tilde{\boldsymbol{y}}^{i} \boldsymbol{y}^{j}\right) g_{\delta q \delta q}^{a}
\end{aligned}
$$

Decompositions analogous to (2.9) to (2.11) hold for antiquarks and for flavor interference distributions.

Corresponding definitions apply for two partons in a left-moving proton, with + and components interchanged in (2.3) to (2.7). Note that the covariant expression of the twodimensional antisymmetric tensor in terms of the four-dimensional one is $\epsilon^{i j}=\epsilon^{+-i j}$ (with $\left.\epsilon_{0123}=1\right)$. In the analogs of $(2.9)$ to $(2.11)$ for left-moving partons one hence needs to change the sign of $\tilde{\boldsymbol{y}}$ and of the pseudoscalar functions $g$ (which can be written as $\epsilon^{i j}$ contracted with a parity even tensor constructed from $\boldsymbol{z}_{1}, \boldsymbol{z}_{2}$ and $\boldsymbol{y}$ ).

All distributions discussed so far allow for two color structures, one where the two fields in the operator $\mathcal{O}_{a_{i}}$ are coupled to a color singlet and one where they are coupled to a color octet. This requires a further index on all distributions, which we will not display in the present work for brevity.

\subsection{Reference frames}

Let us now introduce the reference frames and coordinate axes needed to describe the angular dependence of the cross section.

In the $p p$ center-of-mass we have the $z$ axis pointing along the momentum $p$. The four-vector defining this axis is hence $Z^{\mu}=(p-\bar{p})^{\mu} / \sqrt{2 p \bar{p}}$, where here and in the following we neglect the proton mass. We choose a fixed four-vector $X^{\mu}$ orthogonal to $p$ and $\bar{p}$ to define the $x$ axis. The precise choice does not matter for our purpose, but one may for instance adopt the convention to have the $x$ direction point towards the center of the LHC ring. The $y$ axis is then defined such as to obtain a right-handed coordinate system; the corresponding four-vector can be written as $Y^{\mu}=\epsilon^{\mu}{ }_{\nu \rho \sigma} X^{\nu} \bar{p}^{\rho} p^{\sigma} /(p \bar{p})$.

The kinematics of the gauge boson decays into lepton pairs is conveniently described in the rest frame of the respective gauge boson. The $z$ axis in the rest frame of the boson $V_{i}$ is defined by the four-vector

$$
Z_{i}^{\mu}=\frac{1}{2} \sqrt{Q_{i}^{2}+\boldsymbol{q}_{i}^{2}}\left[\frac{p^{\mu}}{p q_{i}}-\frac{\bar{p}^{\mu}}{\bar{p} q_{i}}\right],
$$




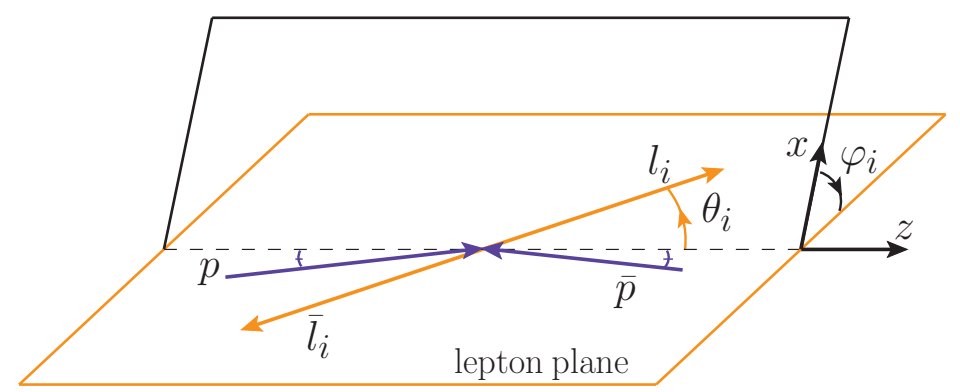

Figure 2. Coordinate system in the rest frame of vector boson $V_{i}$. The $z$ axis bisects the angle between the spatial components of the momenta $p$ and $-\bar{p}$, and the $x$ axis corresponds to a fixed reference direction as explained in the text. (In general, the proton momenta are therefore not in the $x-z$ plane.) $l_{i}$ and $\bar{l}_{i}$ are the momenta of the lepton and the antilepton from the boson decay, respectively. $\theta_{i}$ denotes the polar and $\varphi_{i}$ the azimuthal angle of the lepton. Note that $\varphi_{i}$ is negative in this example.

where $\boldsymbol{q}_{i}$ is the transverse boson momentum in the $p p$ center-of-mass as before. As illustrated for one boson in figure 2, the $z$ axis bisects the angle between the spatial components of $p$ and $-\bar{p}$ in the boson rest frame. The $x$ axis is specified by

$$
X_{i}^{\mu}=\frac{1}{\sqrt{1+\left(X q_{i}\right)^{2} / Q_{i}^{2}}}\left[X^{\mu}-\frac{X q_{i}}{Q_{i}^{2}} q_{i}^{\mu}\right]
$$

and the $y$ axis is again defined so as to obtain a right-handed coordinate system, i.e. by $Y_{i}^{\mu}=\epsilon_{\nu \rho \sigma}^{\mu} Z_{i}^{\nu} X_{i}^{\rho} q_{i}^{\sigma} / Q_{i}$. With these reference axes we define the polar and azimuthal angles $\theta_{i}$ and $\varphi_{i}$ of the lepton (as opposed to the antilepton) in the decay of $V_{i}$, i.e. of $\ell^{-}$ in the decay of a $\gamma^{*}, Z$ or $W^{-}$and of $\nu_{\ell}$ in the decay of a $W^{+}$.

Noting that $X q_{i}$ is the $x$ component of $q_{i}$ in the $p p$ center-of-mass, we see in (2.13) that $X_{1}, X_{2}$ and $X$ differ from each other by amounts of order $\left|\boldsymbol{q}_{i}\right| / Q_{i}$, which is a small parameter in our calculation of the cross section. Likewise, one finds differences of order $\left|\boldsymbol{q}_{i}\right| / Q_{i}$ between $Y_{1}, Y_{2}$ and $Y$. As we shall see shortly, this greatly simplifies the discussion of azimuthal angles in our calculation.

Readers familiar with the analysis of single Drell-Yan production will recognize that our choice of $z$ axes is the same as in the Collins-Soper frame [61]. Useful information about this frame can e.g. be found in $[62,63]$. By contrast, we define $x$ axes (and thus the azimuthal angles $\varphi_{i}$ ) starting from a fixed direction in space, whereas in the Collins-Soper frame the $x$ axis is defined such that the proton momenta lie in the $x-z$ plane. The latter choice becomes undefined when the transverse boson momentum in the $p p$ center-of-mass goes to zero. For unpolarized single Drell-Yan production this is not a problem because in this limiting case all azimuthal dependence in the cross section must vanish due to rotation invariance. However, in the double Drell-Yan process there can be an azimuthal dependence even if $\boldsymbol{q}_{1}$ or $\boldsymbol{q}_{2}$ or both go to zero, as we shall see. Choosing one of these vectors (or any 
linear combination of them) to define $x$ axes would therefore entail ill-defined azimuthal angles at some point in phase space where there can be a nontrivial azimuthal dependence.

The physical cross section must of course not depend on the arbitrary fixed direction specified by $X^{\mu}$. To understand how this happens, we anticipate that our results will depend only on the difference of azimuthal angles whose definition depends on $X^{\mu}$, such as for instance $\varphi_{1}-\varphi_{2}$. These angles are defined in different frames, but to the accuracy of our calculation we can replace them with the azimuthal angles of the leptons in the $p p$ center-of-mass. This is easily seen by writing trigonometric functions of $\varphi_{i}$ in terms of invariant products $X_{i} l_{i}$ and $Y_{i} l_{i}$, where $l_{i}$ is the four-momentum of the lepton from the decay of $V_{i}$. When calculating the cross section we neglect terms of order $\left|\boldsymbol{q}_{i}\right| / Q_{i}$ and can hence approximate $X_{i} l_{i} \approx X l_{i}$ and $Y_{i} l_{i} \approx Y l_{i}$, which gives azimuthal angles in the $p p$ center-of-mass as announced.

\subsection{Hard-scattering cross sections}

If we restore labels for quarks and antiquarks, their flavor and their polarization, the cross section formula (2.1) reads

$$
\begin{aligned}
\frac{d \sigma}{\prod_{i=1}^{2} d x_{i} d \bar{x}_{i} d^{2} \boldsymbol{q}_{i} d \Omega_{i}}= & \frac{1}{C} \sum_{a_{1} a_{2} a_{3} a_{4}} \int \frac{d^{2} \boldsymbol{z}_{1}}{(2 \pi)^{2}} \frac{d^{2} \boldsymbol{z}_{2}}{(2 \pi)^{2}} e^{-i \boldsymbol{z}_{1} \boldsymbol{q}_{1}-i \boldsymbol{z}_{2} \boldsymbol{q}_{2}} \int d^{2} \boldsymbol{y} \\
& \times\left[\frac{d \hat{\sigma}_{a_{1} \bar{a}_{3}}}{d \Omega_{1}} \frac{d \hat{\sigma}_{a_{2} \bar{a}_{4}}}{d \Omega_{2}} F_{a_{1} a_{2}} \bar{F}_{\bar{a}_{3} \bar{a}_{4}}+\frac{d \hat{\sigma}_{a_{1} \bar{a}_{3}}}{d \Omega_{1}} \frac{d \hat{\sigma}_{\bar{a}_{2} a_{4}}}{d \Omega_{2}} F_{a_{1} \bar{a}_{2}} \bar{F}_{\bar{a}_{3} a_{4}}\right. \\
& \left.+\frac{d \hat{\sigma}_{\bar{a}_{1} a_{3}}}{d \Omega_{1}} \frac{d \hat{\sigma}_{a_{2} \bar{a}_{4}}}{d \Omega_{2}} F_{\bar{a}_{1} a_{2}} \bar{F}_{a_{3} \bar{a}_{4}}+\frac{d \hat{\sigma}_{\bar{a}_{1} a_{3}}}{d \Omega_{1}} \frac{d \hat{\sigma}_{\bar{a}_{2} a_{4}}}{d \Omega_{2}} F_{\bar{a}_{1} \bar{a}_{2}} \bar{F}_{a_{3} a_{4}}\right] \\
& +\{\text { flavor interference }\}
\end{aligned}
$$

where here and in the following we omit the label "DPS" for double parton scattering. In all terms the DPDs have arguments as in (2.1), which will be omitted henceforth for brevity. To distinguish the distributions for the left- and right-moving proton we use the notation $F$ and $\bar{F}$ and a corresponding notation for the scalar and pseudoscalar functions $f, \bar{f}$ and $g, \bar{g}$ introduced in section 2.1. The first subscript in $d \sigma_{a_{i} \bar{a}_{j}}$ and $d \sigma_{\bar{a}_{i} a_{j}}$ denotes the right-moving parton and the second subscript the left-moving one. The sum over $a_{1}$ to $a_{4}$ runs over quark flavors and polarizations $(q, \Delta q, \delta q)$.

The flavor interference terms involve the interference DPDs in (2.6) and corresponding interference terms for the hard scattering. These interference terms only appear if the produced bosons are both neutral or both charged, otherwise the quark and antiquark flavors in the annihilation processes do not match. We will return to this in the next section.

Labels for the color structure of the DPDs are not displayed in (2.14). With the conventions of [40], each factor $F \bar{F}$ is to be replaced with the $\operatorname{sum}{ }^{1} F^{1} \bar{F}+{ }^{8} F^{8} \bar{F}$ of color singlet and color octet distributions, without change in the hard-scattering cross sections. This holds for the production of arbitrary color-neutral states in the hard-scattering processes. 
It is straightforward to compute the tree-level cross section for quark-antiquark annihilation into a gauge boson followed by its leptonic decay. In accordance with the power counting scheme underlying the cross section formula (2.14), the transverse boson momenta $\boldsymbol{q}_{i}$ are set to zero in this calculation since by assumption they are small compared with the invariant mass $Q_{i}$. This also simplifies the kinematics of the gauge boson decays as we already noticed in section 2.2 .

Consider first the case where both quark and antiquark are unpolarized or longitudinally polarized. The angular dependence of the cross section is then of the form

$$
\frac{d \hat{\sigma}_{a_{i} \bar{a}_{j}}}{d \Omega_{i}}=\left(1+\cos ^{2} \theta_{i}\right) K_{a_{i} \bar{a}_{j}}\left(Q_{i}\right)+2 \cos \theta_{i} K_{a_{i} \bar{a}_{j}}^{\prime}\left(Q_{i}\right),
$$

with $a_{i}=q_{i}, \Delta q_{i}$ and $\bar{a}_{j}=\bar{q}_{j}, \Delta \bar{q}_{j}$. The integration element reads $d \Omega_{i}=d \varphi_{i} d \cos \theta_{i}$ as usual. The factors $K$ and $K^{\prime}$ depend on coupling constants and on $Q_{i}$ via the gauge boson propagators. One easily finds

$$
K_{\Delta q_{i} \Delta \bar{q}_{j}}=-K_{q_{i} \bar{q}_{j}}, \quad K_{q_{i} \Delta \bar{q}_{j}}=-K_{\Delta q_{i} \bar{q}_{j}}
$$

and analogous relations for $K^{\prime}$, so that

$$
\frac{d \hat{\sigma}_{q_{i} \bar{q}_{j}}}{d \Omega_{i}}=-\frac{d \hat{\sigma}_{\Delta q_{i} \Delta \bar{q}_{j}}}{d \Omega_{i}}, \quad \frac{d \hat{\sigma}_{q_{i} \Delta \bar{q}_{j}}}{d \Omega_{i}}=-\frac{d \hat{\sigma}_{\Delta q_{i} \bar{q}_{j}}}{d \Omega_{i}}
$$

Because of chirality conservation for massless quarks one has vanishing parton-level cross sections for the annihilation of a transversely polarized parton with an unpolarized or longitudinally polarized one, $d \hat{\sigma}_{\delta q_{i} \bar{q}_{j}}=d \hat{\sigma}_{\delta q_{i} \Delta \bar{q}_{j}}=d \hat{\sigma}_{q_{i} \delta \bar{q}_{j}}=d \hat{\sigma}_{\Delta q_{i} \delta \bar{q}_{j}}=0$. If both quark and antiquark are transversely polarized, one finds

$$
\begin{aligned}
\frac{d \hat{\sigma}_{\delta q_{i} \delta \bar{q}_{j}}^{k l}}{d \Omega_{i}}=\sin ^{2} \theta_{i} & \left\{\left[\cos \left(2 \varphi_{i}\right) K_{\delta q_{i} \delta \bar{q}_{j}}\left(Q_{i}\right)-\sin \left(2 \varphi_{i}\right) K_{\delta q_{i} \delta \bar{q}_{j}}^{\prime}\left(Q_{i}\right)\right]\left(\boldsymbol{X}^{k} \boldsymbol{X}^{l}-\boldsymbol{Y}^{k} \boldsymbol{Y}^{l}\right)\right. \\
+ & {\left.\left[\sin \left(2 \varphi_{i}\right) K_{\delta q_{i} \delta \bar{q}_{j}}\left(Q_{i}\right)+\cos \left(2 \varphi_{i}\right) K_{\delta q_{i} \delta \bar{q}_{j}}^{\prime}\left(Q_{i}\right)\right]\left(\boldsymbol{X}^{k} \boldsymbol{Y}^{l}+\boldsymbol{Y}^{k} \boldsymbol{X}^{l}\right)\right\} }
\end{aligned}
$$

with $X$ and $Y$ as defined in section 2.2. The transverse indices $k, l$ in (2.18) refer to the $p p$ center-of-mass, where they are to be contracted with the corresponding indices of the DPDs. We note that contraction of (2.18) with the transverse spin vectors $\boldsymbol{s}^{k}, \overline{\boldsymbol{s}}^{l}$ of the quark and the antiquark gives the simple expression

$$
\frac{d \hat{\sigma}_{\delta q_{i} \delta \bar{q}_{j}}^{k l}}{d \Omega_{i}} s^{k} \bar{s}^{l}=\sin ^{2} \theta_{i}\left[\cos \left(\varphi_{s}+\varphi_{\bar{s}}-2 \varphi_{i}\right) K_{\delta q_{i} \delta \bar{q}_{j}}+\sin \left(\varphi_{s}+\varphi_{\bar{s}}-2 \varphi_{i}\right) K_{\delta q_{i} \delta \bar{q}_{j}}^{\prime}\right],
$$

where $\varphi_{s}$ and $\varphi_{\bar{s}}$ are the azimuthal angles of the spin vectors in the $p p$ center-of-mass and our normalization convention is $s^{2}=\bar{s}^{2}=1$.

The preceding expressions hold for both neutral and charged vector bosons, and the coupling factors $K_{a_{i} \bar{a}_{j}}$ and $K_{a_{i} \bar{a}_{j}}^{\prime}$ appearing in (2.15) and (2.18) are given in appendix A. For neutral boson production the annihilating quark and antiquark have the same flavor. 
In this case we will use $d \hat{\sigma}_{q_{i} \bar{q}_{j}}, d \hat{\sigma}_{q_{i} \Delta \bar{q}_{j}}, \ldots$ with $i \neq j$ to denote the interference terms for flavor $q_{i}$ in the amplitude and flavor $q_{j}$ in the conjugate amplitude. The relations (2.15) to (2.19) remain valid for these interference terms. As can be seen in appendix A, the corresponding coupling factors are complex, and their imaginary parts change sign when the flavor (but not the spin) labels are interchanged, e.g.

$$
K_{q_{1} \bar{q}_{2}}=\left(K_{q_{2} \bar{q}_{1}}\right)^{*}, \quad K_{q_{1} \Delta \bar{q}_{2}}^{\prime}=\left(K_{q_{2} \Delta \bar{q}_{1}}^{\prime}\right)^{*}
$$

We note that for invariant masses $Q_{i}$ far below the $Z$ mass, the neutral boson channel is well approximated by $\gamma^{*}$ production alone. The only nonzero coupling factors in this case are $K_{q_{i} \bar{q}_{j}}=-K_{\Delta q_{i} \Delta \bar{q}_{j}}=K_{\delta q_{i} \delta \bar{q}_{j}}$.

For $W$ boson production we use $d \hat{\sigma}_{q_{i} \bar{q}_{j}}, d \hat{\sigma}_{q_{i} \Delta \bar{q}_{j}}$ etc. to denote cross sections with different flavors $q_{i}, q_{j}$ in the initial state. We do not need a separate notation for flavor interference terms in this case, because the product $d \hat{\sigma}_{a_{1} \bar{a}_{3}} d \hat{\sigma}_{a_{2} \bar{a}_{4}}$ of cross sections for $W W$ production is equal to the product of the corresponding interference terms, except for CKM factors that can easily be identified. Using that $W$ bosons only couple to left-handed fermions, we find further simplifications for the coupling factors:

$$
K_{q_{i} \bar{q}_{j}}=K_{q_{i} \Delta \bar{q}_{j}}, \quad K_{\delta q_{i} \delta \bar{q}_{j}}=0, \quad K_{a_{1} \bar{a}_{2}}^{\prime}=K_{a_{1} \bar{a}_{2}},
$$

where the second relation reflects that the operator $\mathcal{O}_{\delta q}$ for transverse quark polarization corresponds to the interference between left- and right-handed quarks. Together with the relations (2.16) we are thus left with only one independent coupling factor for $W^{-}$and only one for $W^{+}$production.

So far we have discussed cross sections and interference terms $d \hat{\sigma}_{a_{i} \bar{a}_{j}}$ for the annihilation of a right-moving quark with a left-moving antiquark. The cross sections and interference terms $d \hat{\sigma}_{\bar{a}_{j} a_{i}}$ for right-moving antiquarks and left-moving quarks have the same form as in (2.15) and (2.18). The associated coupling factors are given by

$$
K_{\bar{q}_{j} q_{i}}=\left(K_{q_{i} \bar{q}_{j}}\right)^{*}, \quad K_{\bar{q}_{j} q_{i}}^{\prime}=-\left(K_{q_{i} \bar{q}_{j}}^{\prime}\right)^{*}
$$

and analogous relations for the spin combinations $\Delta q \Delta q$ and $\delta q \delta q$, and by

$$
K_{\bar{q}_{j} \Delta q_{i}}=-\left(K_{q_{i} \Delta \bar{q}_{j}}\right)^{*}, \quad \quad K_{\bar{q}_{j} \Delta q_{i}}^{\prime}=\left(K_{q_{i} \Delta \bar{q}_{j}}^{\prime}\right)^{*}
$$

and an analogous relation for the spin combination $\Delta q q$.

\section{The double Drell-Yan cross section}

Inserting the hard-scattering cross sections (2.15), (2.18) and the DPD decompositions (2.9) to (2.11) into the factorization formula (2.14), we obtain our final results for the double parton scattering contribution to four-lepton production. 
For the production and decay of two $W$ bosons, the result has a simple structure thanks to the relations $(2.21)$,

$$
\begin{aligned}
& \frac{d \sigma^{W W}}{\prod_{i=1}^{2} d x_{i} d \bar{x}_{i} d^{2} \boldsymbol{q}_{i} d \Omega_{i}}=\frac{1}{C} \sum_{q_{1} q_{2} q_{3} q_{4}} K_{q_{1} \bar{q}_{3}}\left(Q_{1}\right) K_{q_{2} \bar{q}_{4}}\left(Q_{2}\right) \int \frac{d^{2} \boldsymbol{z}_{1}}{(2 \pi)^{2}} \frac{d^{2} \boldsymbol{z}_{2}}{(2 \pi)^{2}} e^{-i \boldsymbol{z}_{1} \boldsymbol{q}_{1}-i \boldsymbol{z}_{2} \boldsymbol{q}_{2}} \int d^{2} \boldsymbol{y} \\
& \times\left[\left(1+\cos \theta_{1}\right)^{2}\left(1+\cos \theta_{2}\right)^{2}\right. \\
& \times\left(f_{q_{1} q_{2}}+f_{\Delta q_{1} \Delta q_{2}}-g_{q_{1} \Delta q_{2}}-g_{\Delta q_{1} q_{2}}\right)\left(\bar{f}_{\bar{q}_{3} \bar{q}_{4}}+\bar{f}_{\Delta \bar{q}_{3} \Delta \bar{q}_{4}}-\bar{g}_{\bar{q}_{3} \Delta \bar{q}_{4}}-\bar{g}_{\Delta \bar{q}_{3} \bar{q}_{4}}\right) \\
& +\left(1+\cos \theta_{1}\right)^{2}\left(1-\cos \theta_{2}\right)^{2} \\
& \times\left(f_{q_{1} \bar{q}_{4}}-f_{\Delta q_{1} \Delta \bar{q}_{4}}+g_{q_{1} \Delta \bar{q}_{4}}-g_{\Delta q_{1} \bar{q}_{4}}\right)\left(\bar{f}_{\bar{q}_{3} q_{2}}-\bar{f}_{\Delta \bar{q}_{3} \Delta q_{2}}+\bar{g}_{\bar{q}_{3} \Delta q_{2}}-\bar{g}_{\Delta \bar{q}_{3} q_{2}}\right) \\
& +\left(1-\cos \theta_{1}\right)^{2}\left(1+\cos \theta_{2}\right)^{2} \\
& \times\left(f_{\bar{q}_{3} q_{2}}-f_{\Delta \bar{q}_{3} \Delta q_{2}}-g_{\bar{q}_{3} \Delta q_{2}}+g_{\Delta \bar{q}_{3} q_{2}}\right)\left(\bar{f}_{q_{1} \bar{q}_{4}}-\bar{f}_{\Delta q_{1} \Delta \bar{q}_{4}}-\bar{g}_{q_{1} \Delta \bar{q}_{4}}+\bar{g}_{\Delta q_{1} \bar{q}_{4}}\right) \\
& +\left(1-\cos \theta_{1}\right)^{2}\left(1-\cos \theta_{2}\right)^{2} \\
& \times\left(f_{\bar{q}_{3} \bar{q}_{4}}+f_{\Delta \bar{q}_{3} \Delta \bar{q}_{4}}+g_{\bar{q}_{3} \Delta \bar{q}_{4}}+g_{\Delta \bar{q}_{3} \bar{q}_{4}}\right)\left(\bar{f}_{q_{1} q_{2}}+\bar{f}_{\Delta q_{1} \Delta q_{2}}+\bar{g}_{q_{1} \Delta q_{2}}+\bar{g}_{\Delta q_{1} q_{2}}\right) \\
& +\{\text { flavor interference }\}] \text {, }
\end{aligned}
$$

where the sum over $q_{1}$ to $q_{4}$ runs over quark flavors. The flavor interference terms are obtained by replacing the DPDs in one or in both protons with their interference analogs and by appropriately changing the CKM factors in the product $K_{q_{1} \bar{q}_{3}} K_{q_{2} \bar{q}_{4}}$. Different types of flavor interference terms are shown in figure 3. Taking into account the minus sign in the definition of pseudoscalar distributions for left-moving partons, e.g. in $\bar{F}_{q_{i} \Delta \bar{q}_{j}}=-\bar{g}_{q_{i} \Delta \bar{q}_{j}}$, we recognize that the DPD combinations in (3.1) correspond to negativehelicity quarks and positive-helicity antiquarks, as required by the left-handed nature of the charged weak current.

We see that for $W$ pair production the presence of longitudinal parton spin correlations in the proton changes the overall rate of the cross section as well as the distribution in the polar angles of the decay leptons.

For one or two neutral bosons $\left(\gamma^{*}, Z\right)$ the structure of the cross section is more complicated. We split the cross section (2.14) into three parts, $\sigma^{(0)}$ for the case without transverse quark polarization and $\sigma^{(1)}, \sigma^{(2)}$ for the cases where one or two hard interactions are initiated by transversely polarized quarks. The contribution with only unpolarized and longitudinally polarized partons reads

$$
\begin{aligned}
& \frac{d \sigma^{(0)}}{\prod_{i=1}^{2} d x_{i} d \bar{x}_{i} d^{2} \boldsymbol{q}_{i} d \Omega_{i}}=\frac{1}{C} \sum_{q_{1} q_{2} q_{3} q_{4}} \int \frac{d^{2} \boldsymbol{z}_{1}}{(2 \pi)^{2}} \frac{d^{2} \boldsymbol{z}_{2}}{(2 \pi)^{2}} e^{-i \boldsymbol{z}_{1} \boldsymbol{q}_{1}-i \boldsymbol{z}_{2} \boldsymbol{q}_{2}} \int d^{2} \boldsymbol{y} \\
& \times\{[\left.\left(1+\cos ^{2} \theta_{1}\right) K_{q_{1} \bar{q}_{3}}+2 \cos \theta_{1} K_{q_{1} \bar{q}_{3}}^{\prime}\right]\left[\left(1+\cos ^{2} \theta_{2}\right) K_{q_{2} \bar{q}_{4}}+2 \cos \theta_{2} K_{q_{2} \bar{q}_{4}}^{\prime}\right] \\
& \quad \times\left(f_{q_{1} q_{2}} \bar{f}_{\bar{q}_{3} \bar{q}_{4}}+f_{\Delta q_{1} \Delta q_{2}} \bar{f}_{\Delta \bar{q}_{3} \Delta \bar{q}_{4}}+g_{q_{1} \Delta q_{2}} \bar{g}_{\bar{q}_{3} \Delta \bar{q}_{4}}+g_{\Delta q_{1} q_{2}} \bar{g}_{\Delta \bar{q}_{3} \bar{q}_{4}}\right) \\
&+\left[\left(1+\cos ^{2} \theta_{1}\right) K_{q_{1} \Delta \bar{q}_{3}}+2 \cos \theta_{1} K_{q_{1} \Delta \bar{q}_{3}}^{\prime}\right]\left[\left(1+\cos ^{2} \theta_{2}\right) K_{q_{2} \Delta \bar{q}_{4}}+2 \cos \theta_{2} K_{q_{2} \Delta \bar{q}_{4}}^{\prime}\right] \\
& \quad \times\left(f_{q_{1} q_{2}} \bar{f}_{\Delta \bar{q}_{3} \Delta \bar{q}_{4}}+f_{\Delta q_{1} \Delta q_{2}} \bar{f}_{\bar{q}_{3} \bar{q}_{4}}+g_{q_{1} \Delta q_{2}} \bar{g}_{\Delta \bar{q}_{3} \bar{q}_{4}}+g_{\Delta q_{1} q_{2}} \bar{g}_{\bar{q}_{3} \Delta \bar{q}_{4}}\right)
\end{aligned}
$$




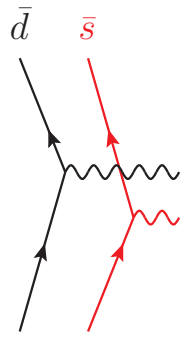

u c

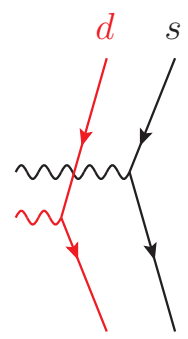

$\bar{u} \quad \bar{c}$

a

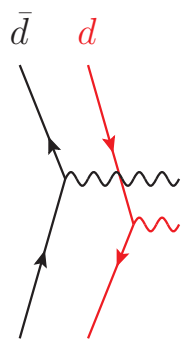

$u \quad \bar{u}$

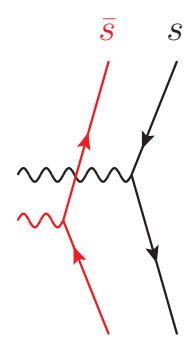

c $\bar{c}$

c

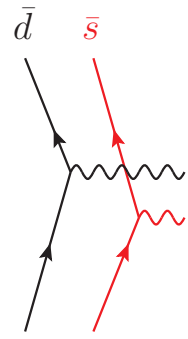

u c

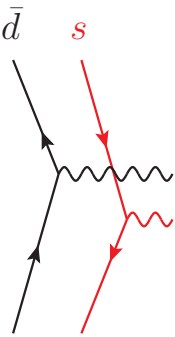

$u \quad \bar{u}$

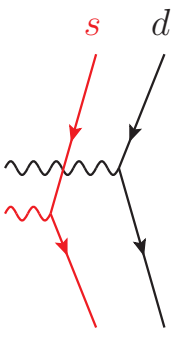

$\bar{u} \quad \bar{c}$

b

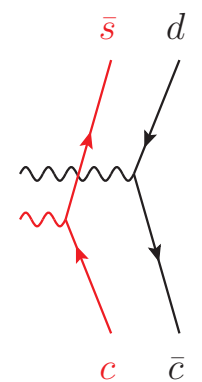

d

Figure 3. Hard-scattering graphs for the production of $W^{+} W^{+}(\mathrm{a}, \mathrm{b})$ or of $W^{+} W^{-}(\mathrm{c}, \mathrm{d})$. The labels $q$ and $\bar{q}$ indicate whether a parton corresponds to a quark field or a conjugate quark field in the relevant DPD. Graphs (b) and (d) are multiplied with interference distributions for one of the protons, whereas graphs (a) and (c) go along with interference distributions for both protons.

$$
\begin{aligned}
- & {\left[\left(1+\cos ^{2} \theta_{1}\right) K_{q_{1} \bar{q}_{3}}+2 \cos \theta_{1} K_{q_{1} \bar{q}_{3}}^{\prime}\right]\left[\left(1+\cos ^{2} \theta_{2}\right) K_{q_{2} \Delta \bar{q}_{4}}+2 \cos \theta_{2} K_{q_{2} \Delta \bar{q}_{4}}^{\prime}\right] } \\
& \times\left(g_{q_{1} \Delta q_{2}} \bar{f}_{\bar{q}_{3} \bar{q}_{4}}+g_{\Delta q_{1} q_{2}} \bar{f}_{\Delta \bar{q}_{3} \Delta \bar{q}_{4}}+f_{q_{1} q_{2}} \bar{g}_{\bar{q}_{3} \Delta \bar{q}_{4}}+f_{\Delta q_{1} \Delta q_{2}} \bar{g}_{\Delta \bar{q}_{3} \bar{q}_{4}}\right) \\
- & {\left[\left(1+\cos ^{2} \theta_{1}\right) K_{q_{1} \Delta \bar{q}_{3}}+2 \cos \theta_{1} K_{q_{1} \Delta \bar{q}_{3}}^{\prime}\right]\left[\left(1+\cos ^{2} \theta_{2}\right) K_{q_{2} \bar{q}_{4}}+2 \cos \theta_{2} K_{q_{2} \bar{q}_{4}}^{\prime}\right] } \\
& \left.\times\left(g_{q_{1} \Delta q_{2}} \bar{f}_{\Delta \bar{q}_{3} \Delta \bar{q}_{4}}+g_{\Delta q_{1} q_{2}} \bar{f}_{\bar{q}_{3} \bar{q}_{4}}+f_{q_{1} q_{2}} \bar{g}_{\Delta \bar{q}_{3} \bar{q}_{4}}+f_{\Delta q_{1} \Delta q_{2}} \bar{g}_{\bar{q}_{3} \Delta \bar{q}_{4}}\right)\right\} \\
+ & \text { flavor interference }\}+\{q \bar{q} \text { permutations }\} .
\end{aligned}
$$

The $q \bar{q}$ permutation terms are obtained by permutation of the quark-antiquark assignments in the DPDs and in the coupling factors $K, K^{\prime}$ as specified in (2.14). For neutral bosons the annihilating quark and antiquark have the same flavor, i.e. one has $q_{1}=q_{3}\left(q_{2}=q_{4}\right)$ if $V_{1}\left(V_{2}\right)$ is neutral. The flavor interference term for neutral boson pairs is then obtained by replacing all distributions $f, g, \bar{f}, \bar{g}$ with their interference analogs $f^{I}, g^{I}, \bar{f}^{I}, \bar{g}^{I}$ and by interchanging $1 \leftrightarrow 2$ in the second subscript of the coupling factors, e.g. $K_{q_{1} \bar{q}_{1}} K_{q_{2} \Delta \bar{q}_{2}}^{\prime} \rightarrow$ $K_{q_{1} \bar{q}_{2}} K_{q_{2} \Delta \bar{q}_{1}}^{\prime}$. The relations (2.8), (2.20) and their analogs for other polarizations ensure that the sum over all flavor assignments in (3.2) gives a real-valued cross section. Example graphs for flavor interference are shown in figure 4.

We see in (3.2) that longitudinal parton spin correlations change the overall rate of double parton scattering and the dependence on the polar angles of the leptons, due to the differences between the coupling factors $K_{q_{i} \bar{q}_{j}}, K_{q_{i} \bar{q}_{j}}^{\prime}$ and $K_{q_{i} \Delta \bar{q}_{j}}, K_{q_{i} \Delta \bar{q}_{j}}^{\prime}$. Only in the 




u d

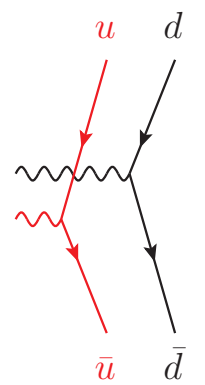

a

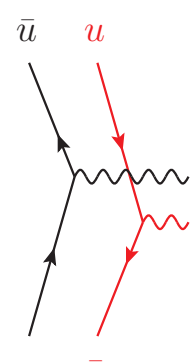

$u \quad \bar{u}$

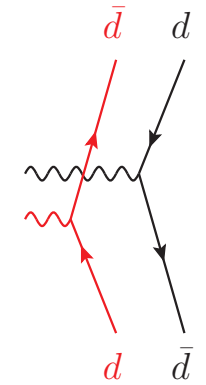

b

Figure 4. Hard-scattering graphs for the production of two neutral gauge bosons. The labels $q$ and $\bar{q}$ have the same meaning as in figure 3 .

neutral boson channel at $Q_{i}$ values small enough to neglect $Z$ production does one have a fixed angular dependence $d \sigma^{(0)} / d \cos \theta_{i} \propto 1+\cos ^{2} \theta_{i}$.

We now turn towards the part of the cross section where one of the two annihilation processes involves transverse quark polarization (and thus produces a neutral gauge boson). It reads

$$
\begin{aligned}
& \frac{d \sigma^{(1)}}{\prod_{i=1}^{2} d x_{i} d \bar{x}_{i} d^{2} \boldsymbol{q}_{i} d \Omega_{i}}=\frac{1}{C} \sin ^{2} \theta_{2} \sum_{q_{1} q_{2} q_{3}} \int \frac{d^{2} \boldsymbol{z}_{1}}{(2 \pi)^{2}} \frac{d^{2} \boldsymbol{z}_{2}}{(2 \pi)^{2}} e^{-i \boldsymbol{z}_{1} \boldsymbol{q}_{1}-i \boldsymbol{z}_{2} \boldsymbol{q}_{2}} \int d^{2} \boldsymbol{y} \boldsymbol{y}^{2} M^{2} \\
& \times(\left.\left(1+\cos ^{2} \theta_{1}\right) K_{q_{1} \bar{q}_{3}}+2 \cos \theta_{1} K_{q_{1} \bar{q}_{3}}^{\prime}\right] \\
& \times\left\{\left[\cos 2\left(\varphi_{2}-\varphi_{y}\right) K_{\delta q_{2} \delta \bar{q}_{2}}-\sin 2\left(\varphi_{2}-\varphi_{y}\right) K_{\delta q_{2} \delta \bar{q}_{2}}^{\prime}\right]\right. \\
& \times\left(f_{q_{1} \delta q_{2}} \bar{f}_{\bar{q}_{3} \delta \bar{q}_{2}}-g_{q_{1} \delta q_{2}} \bar{g}_{\bar{q}_{3} \delta \bar{q}_{2}}-f_{\Delta q_{1} \delta q_{2}} \bar{f}_{\Delta \bar{q}_{3} \delta \bar{q}_{2}}+g_{\Delta q_{1} \delta q_{2}} \bar{g}_{\Delta \bar{q}_{3} \delta \bar{q}_{2}}\right) \\
&+ {\left[\sin 2\left(\varphi_{2}-\varphi_{y}\right) K_{\delta q_{2} \delta \bar{q}_{2}}+\cos 2\left(\varphi_{2}-\varphi_{y}\right) K_{\delta q_{2} \delta \bar{q}_{2}}^{\prime}\right] } \\
&\left.\times\left(f_{q_{1} \delta q_{2}} \bar{g}_{\bar{q}_{3} \delta \bar{q}_{2}}+g_{q_{1} \delta q_{2}} \bar{f}_{\bar{q}_{3} \delta \bar{q}_{2}}+f_{\Delta q_{1} \delta q_{2}} \bar{g}_{\Delta \bar{q}_{3} \delta \bar{q}_{2}}+g_{\Delta q_{1} \delta q_{2}} \bar{f}_{\Delta \bar{q}_{3} \delta \bar{q}_{2}}\right)\right\} \\
&-[\left.\left(1+\cos { }^{2} \theta_{1}\right) K_{q_{1} \Delta \bar{q}_{3}}+2 \cos \theta_{1} K_{q_{1} \Delta \bar{q}_{3}}^{\prime}\right] \\
& \times\{ {\left[\cos 2\left(\varphi_{2}-\varphi_{y}\right) K_{\delta q_{2} \delta \bar{q}_{2}}-\sin 2\left(\varphi_{2}-\varphi_{y}\right) K_{\delta q_{2} \delta \bar{q}_{2}}^{\prime}\right] } \\
& \times\left(f_{q_{1} \delta q_{2}} \bar{g}_{\Delta \bar{q}_{3} \delta \bar{q}_{2}}-g_{q_{1} \delta q_{2}} \bar{f}_{\Delta \bar{q}_{3} \delta \bar{q}_{2}}-f_{\Delta q_{1} \delta q_{2}} \bar{g}_{\bar{q}_{3} \delta \bar{q}_{2}}+g_{\Delta q_{1} \delta q_{2}} \bar{f}_{\bar{q}_{3} \delta \bar{q}_{2}}\right) \\
&+ {\left[\sin 2\left(\varphi_{2}-\varphi_{y}\right) K_{\delta q_{2} \delta \bar{q}_{2}}+\cos 2\left(\varphi_{2}-\varphi_{y}\right) K_{\delta q_{2} \delta \bar{q}_{2}}^{\prime}\right] } \\
&\left.\left.\times\left(f_{q_{1} \delta q_{2}} \bar{f}_{\Delta \bar{q}_{3} \delta \bar{q}_{2}}+g_{q_{1} \delta q_{2}} \bar{g}_{\Delta \bar{q}_{3} \delta \bar{q}_{2}}+f_{\Delta q_{1} \delta q_{2}} \bar{f}_{\bar{q}_{3} \delta \bar{q}_{2}}+g_{\Delta q_{1} \delta q_{2}} \bar{g}_{\bar{q}_{3} \delta \bar{q}_{2}}\right)\right\}\right) \\
&+\{\text { flavor interference }\}+\{q \bar{q} \operatorname{permutations}\}+\{\text { transv. pol. in interaction } 1\}
\end{aligned}
$$

where the flavor interference and $q \bar{q}$ permutation terms are obtained in the same way as in (3.2). The terms for transverse polarization in interaction 1 are obtained by replacing labels as $1 \rightarrow 2,2 \rightarrow 1,3 \rightarrow 4$ in the coupling factors and by making the same replacement in the DPD subscripts after interchanging their order, i.e. $f_{a_{1} \delta q_{2}} \rightarrow f_{\delta q_{1} a_{2}}, \bar{g}_{\bar{a}_{3} \delta \bar{q}_{2}} \rightarrow \bar{g}_{\delta \bar{q}_{1} \bar{a}_{4}}$ etc. 
The azimuthal angle $\varphi_{2}$ of the lepton produced in interaction 2 has already been defined, and $\varphi_{y}$ is the azimuthal angle of $\boldsymbol{y}$ in the $p p$ center-of-mass. As anticipated in section 2.2, the cross section depends only on the difference $\varphi_{2}-\varphi_{y}$ of these angles, in agreement with rotation invariance. The $\varphi_{y}$ dependence in (3.3) arises from the uncontracted vectors $\boldsymbol{y}$ and $\tilde{\boldsymbol{y}}$ in the DPDs (2.10) for transversely polarized partons: it is hence this polarization which enables a dependence of the cross section on the azimuthal angle of the produced lepton.

The transverse distance $\boldsymbol{y}$ is integrated over in (3.3) and hence not measurable. The $\boldsymbol{y}$ integration is nontrivial because the DPDs depend on the azimuthal angles between $\boldsymbol{y}$ and $\boldsymbol{z}_{1}$ and $\boldsymbol{z}_{2}$, whose directions are in turn correlated with those of $\boldsymbol{q}_{1}$ and $\boldsymbol{q}_{2}$ through the exponential $e^{-i \boldsymbol{z}_{1} \boldsymbol{q}_{1}-i \boldsymbol{z}_{2} \boldsymbol{q}_{2}}$. The integral over $\boldsymbol{y}, \boldsymbol{z}_{1}$ and $\boldsymbol{z}_{2}$ in the cross section thus turns the $\varphi_{y}$ dependence into a dependence on the azimuthal angles of the transverse momenta $\boldsymbol{q}_{1}$ and $\boldsymbol{q}_{2}$. All together we thus see that a correlation between $\boldsymbol{y}$ and the transverse polarization of parton 2 in the DPDs leads to an azimuthal correlation between the lepton from interaction 2 and both transverse vector boson momenta. This is similar (but not identical) to single Drell-Yan production, where a correlation between the transverse polarization of a parton and its transverse momentum induces an azimuthal correlation between the momenta of the vector boson and its decay lepton [64].

We finally turn to the case where both vector bosons are produced from transversely polarized quarks. The corresponding contribution to the cross section is

$$
\begin{aligned}
& \frac{d \sigma^{(2)}}{\prod_{i=1}^{2} d x_{i} d \bar{x}_{i} d^{2} \boldsymbol{q}_{i} d \Omega_{i}}=\frac{1}{C} 2 \sin ^{2} \theta_{1} \sin ^{2} \theta_{2} \sum_{q_{1} q_{2}} \int \frac{d^{2} \boldsymbol{z}_{1}}{(2 \pi)^{2}} \frac{d^{2} \boldsymbol{z}_{2}}{(2 \pi)^{2}} e^{-i \boldsymbol{z}_{1} \boldsymbol{q}_{1}-i \boldsymbol{z}_{2} \boldsymbol{q}_{2}} \int d^{2} \boldsymbol{y} \\
& \times\left\{\left[\cos 2\left(\varphi_{1}-\varphi_{2}\right)\left(K_{\delta q_{1} \delta \bar{q}_{1}} K_{\delta q_{2} \delta \bar{q}_{2}}+K_{\delta q_{1} \delta \bar{q}_{1}}^{\prime} K_{\delta q_{2} \delta \bar{q}_{2}}^{\prime}\right)\right.\right. \\
& \left.-\sin 2\left(\varphi_{1}-\varphi_{2}\right)\left(K_{\delta q_{1} \delta \bar{q}_{1}}^{\prime} K_{\delta q_{2} \delta \bar{q}_{2}}-K_{\delta q_{1} \delta \bar{q}_{1}} K_{\delta q_{2} \delta \bar{q}_{2}}^{\prime}\right)\right] \\
& \times\left(f_{\delta q_{1} \delta q_{2}} \bar{f}_{\delta \bar{q}_{1} \delta \bar{q}_{2}}-\boldsymbol{y}^{4} M^{4} g_{\delta q_{1} \delta q_{2}}^{a} \bar{g}_{\delta \bar{q}_{1} \delta \bar{q}_{2}}^{a}\right) \\
& +\left[\sin 2\left(\varphi_{1}-\varphi_{2}\right)\left(K_{\delta q_{1} \delta \bar{q}_{1}} K_{\delta q_{2} \delta \bar{q}_{2}}+K_{\delta q_{1} \delta \bar{q}_{1}}^{\prime} K_{\delta q_{2} \delta \bar{q}_{2}}^{\prime}\right)\right. \\
& \left.+\cos 2\left(\varphi_{1}-\varphi_{2}\right)\left(K_{\delta q_{1} \delta \bar{q}_{1}}^{\prime} K_{\delta q_{2} \delta \bar{q}_{2}}-K_{\delta q_{1} \delta \bar{q}_{1}} K_{\delta q_{2} \delta \bar{q}_{2}}^{\prime}\right)\right] \\
& \times \boldsymbol{y}^{2} M^{2}\left(f_{\delta q_{1} \delta q_{2}} \bar{g}_{\delta \bar{q}_{1} \delta \bar{q}_{2}}^{a}+g_{\delta q_{1} \delta q_{2}}^{a} \bar{f}_{\delta \bar{q}_{1} \delta \bar{q}_{2}}\right) \\
& +\left[\cos 2\left(\varphi_{1}+\varphi_{2}-2 \varphi_{y}\right)\left(K_{\delta q_{1} \delta \bar{q}_{1}} K_{\delta q_{2} \delta \bar{q}_{2}}-K_{\delta q_{1} \delta \bar{q}_{1}}^{\prime} K_{\delta q_{2} \delta \bar{q}_{2}}^{\prime}\right)\right. \\
& \left.-\sin 2\left(\varphi_{1}+\varphi_{2}-2 \varphi_{y}\right)\left(K_{\delta q_{1} \delta \bar{q}_{1}}^{\prime} K_{\delta q_{2} \delta \bar{q}_{2}}+K_{\delta q_{1} \delta \bar{q}_{1}} K_{\delta q_{2} \delta \bar{q}_{2}}^{\prime}\right)\right] \\
& \times \boldsymbol{y}^{4} M^{4}\left(f_{\delta q_{1} \delta q_{2}}^{t} \bar{f}_{\delta \bar{q}_{1} \delta \bar{q}_{2}}^{t}-g_{\delta q_{1} \delta q_{2}}^{s} \bar{g}_{\delta \bar{q}_{1} \delta \bar{q}_{2}}^{s}\right) \\
& -\left[\sin 2\left(\varphi_{1}+\varphi_{2}-2 \varphi_{y}\right)\left(K_{\delta q_{1} \delta \bar{q}_{1}} K_{\delta q_{2} \delta \bar{q}_{2}}-K_{\delta q_{1} \delta \bar{q}_{1}}^{\prime} K_{\delta q_{2} \delta \bar{q}_{2}}^{\prime}\right)\right. \\
& \left.+\cos 2\left(\varphi_{1}+\varphi_{2}-2 \varphi_{y}\right)\left(K_{\delta q_{1} \delta \bar{q}_{1}}^{\prime} K_{\delta q_{2} \delta \bar{q}_{2}}+K_{\delta q_{1} \delta \bar{q}_{1}} K_{\delta q_{2} \delta \bar{q}_{2}}^{\prime}\right)\right] \\
& \left.\times \boldsymbol{y}^{4} M^{4}\left(f_{\delta q_{1} \delta q_{2}}^{t} \bar{g}_{\delta \bar{q}_{1} \delta \bar{q}_{2}}^{s}+g_{\delta q_{1} \delta q_{2}}^{s} \bar{f}_{\delta \bar{q}_{1} \delta \bar{q}_{2}}^{t}\right)\right\} \\
& +\{\text { flavor interference }\}+\{q \bar{q} \text { permutations }\}
\end{aligned}
$$


and depends on the azimuthal angles $\varphi_{1}, \varphi_{2}$ and $\varphi_{y}$ in addition to the polar angles $\theta_{1}$ and $\theta_{2}$. The flavor interference and $q \bar{q}$ permutation terms are again obtained as in (3.2).

The terms depending on $\varphi_{1}-\varphi_{2}$ describe a transverse correlation between the leptonic decay planes of the vector bosons. By contrast, the terms with $\varphi_{1}+\varphi_{2}-2 \varphi_{y}$ describe an azimuthal correlation between the lepton momenta and the direction between the hard interactions, which after integration over $\boldsymbol{y}, \boldsymbol{z}_{1}$ and $\boldsymbol{z}_{2}$ turns into an azimuthal correlation between the lepton momenta and the momenta of the two bosons.

\subsection{Cross section integrated over transverse boson momenta}

Integration over the transverse momenta of the two vector bosons yields cross sections expressed in terms of collinear double parton distributions $F\left(x_{1}, x_{2}, \boldsymbol{y}\right)$. Their spin structure is as in (2.9) to (2.11) but without pseudoscalar functions $g$, because one cannot construct a pseudoscalar with only one vector $\boldsymbol{y}$.

Upon integration over $\boldsymbol{q}_{1}$ and $\boldsymbol{q}_{2}$, the cross section (3.1) for $W$ pair production becomes

$$
\begin{aligned}
& \frac{d \sigma^{W W}}{\prod_{i=1}^{2} d x_{i} d \bar{x}_{i} d \Omega_{i}}=\frac{1}{C} \sum_{q_{1} q_{2} q_{3} q_{4}} K_{q_{1} \bar{q}_{3}} K_{q_{2} \bar{q}_{4}} \\
& \quad \times\left\{\left(1+\cos \theta_{1}\right)^{2}\left(1+\cos \theta_{2}\right)^{2} \int d^{2} \boldsymbol{y}\left(f_{q_{1} q_{2}}+f_{\Delta q_{1} \Delta q_{2}}\right)\left(\bar{f}_{\bar{q}_{3} \bar{q}_{4}}+\bar{f}_{\Delta \bar{q}_{3} \Delta \bar{q}_{4}}\right)\right. \\
& \quad+\left(1+\cos \theta_{1}\right)^{2}\left(1-\cos \theta_{2}\right)^{2} \int d^{2} \boldsymbol{y}\left(f_{q_{1} \bar{q}_{4}}-f_{\Delta q_{1} \Delta \bar{q}_{4}}\right)\left(\bar{f}_{\bar{q}_{3} q_{2}}-\bar{f}_{\Delta \bar{q}_{3} \Delta q_{2}}\right) \\
& \quad+\left(1-\cos \theta_{1}\right)^{2}\left(1+\cos \theta_{2}\right)^{2} \int d^{2} \boldsymbol{y}\left(f_{\bar{q}_{3} q_{2}}-f_{\Delta \bar{q}_{3} \Delta q_{2}}\right)\left(\bar{f}_{q_{1} \bar{q}_{4}}-\bar{f}_{\Delta q_{1} \Delta \bar{q}_{4}}\right) \\
& \left.\quad+\left(1-\cos \theta_{1}\right)^{2}\left(1-\cos \theta_{2}\right)^{2} \int d^{2} \boldsymbol{y}\left(f_{\bar{q}_{3} \bar{q}_{4}}+f_{\Delta \bar{q}_{3} \Delta \bar{q}_{4}}\right)\left(\bar{f}_{q_{1} q_{2}}+\bar{f}_{\Delta q_{1} \Delta q_{2}}\right)\right\} \\
& \quad+\{\text { flavor interference }\},
\end{aligned}
$$

where the arguments of the distributions are $f\left(x_{1}, x_{2}, \boldsymbol{y}\right)$ and $\bar{f}\left(\bar{x}_{1}, \bar{x}_{2}, \boldsymbol{y}\right)$. In the general case we have a contribution

$$
\begin{aligned}
& \frac{d \sigma^{(0)}}{\prod_{i=1}^{2} d x_{i} d \bar{x}_{i} d \Omega_{i}}=\frac{1}{C} \sum_{q_{1} q_{2} q_{3} q_{4}} \\
& \times\left\{\left[\left(1+\cos ^{2} \theta_{1}\right) K_{q_{1} \bar{q}_{3}}+2 \cos \theta_{1} K_{q_{1} \bar{q}_{3}}^{\prime}\right]\left[\left(1+\cos ^{2} \theta_{2}\right) K_{q_{2} \bar{q}_{4}}+2 \cos \theta_{2} K_{q_{2} \bar{q}_{4}}^{\prime}\right]\right. \\
& \quad \times \int d^{2} \boldsymbol{y}\left(f_{q_{1} q_{2}} \bar{f}_{\bar{q}_{3} \bar{q}_{4}}+f_{\Delta q_{1} \Delta q_{2}} \bar{f}_{\Delta \bar{q}_{3} \Delta \bar{q}_{4}}\right) \\
& \quad+\left[\left(1+\cos ^{2} \theta_{1}\right) K_{q_{1} \Delta \bar{q}_{3}}+2 \cos \theta_{1} K_{q_{1} \Delta \bar{q}_{3}}^{\prime}\right]\left[\left(1+\cos ^{2} \theta_{2}\right) K_{q_{2} \Delta \bar{q}_{4}}+2 \cos \theta_{2} K_{q_{2} \Delta \bar{q}_{4}}^{\prime}\right] \\
& \left.\quad \times \int d^{2} \boldsymbol{y}\left(f_{q_{1} q_{2}} \bar{f}_{\Delta \bar{q}_{3} \Delta \bar{q}_{4}}+f_{\Delta q_{1} \Delta q_{2}} \bar{f}_{\bar{q}_{3} \bar{q}_{4}}\right)\right\} \\
& +\{\text { flavor interference }\}+\{q \bar{q} \text { permutations }\}
\end{aligned}
$$


from unpolarized and longitudinally polarized partons. The contribution with transverse quark polarization in one of the two hard interactions now vanishes,

$$
\frac{d \sigma^{(1)}}{\prod_{i=1}^{2} d x_{i} d \bar{x}_{i} d \Omega_{i}}=0 .
$$

This is because integration of (3.3) over $\boldsymbol{q}_{1}$ and $\boldsymbol{q}_{2}$ sets $\boldsymbol{z}_{1}=\boldsymbol{z}_{2}=\mathbf{0}$, after which the $\boldsymbol{y}$ integral gives zero due to the azimuthal dependence on $\varphi_{y}$. By contrast, the contribution with transverse quark polarization in both hard interactions remains nonzero,

$$
\begin{aligned}
& \frac{d \sigma^{(2)}}{\prod_{i=1}^{2} d x_{i} d \bar{x}_{i} d \Omega_{i}}=\frac{1}{C} 2 \sin ^{2} \theta_{1} \sin ^{2} \theta_{2} \sum_{q_{1} q_{2}} \\
& \times\left\{\left[\cos 2\left(\varphi_{1}-\varphi_{2}\right)\left(K_{\delta q_{1} \delta \bar{q}_{1}} K_{\delta q_{2} \delta \bar{q}_{2}}+K_{\delta q_{1} \delta \bar{q}_{1}}^{\prime} K_{\delta q_{2} \delta \bar{q}_{2}}^{\prime}\right)\right.\right. \\
& \left.\left.\quad-\sin 2\left(\varphi_{1}-\varphi_{2}\right)\left(K_{\delta q_{1} \delta \bar{q}_{1}}^{\prime} K_{\delta q_{2} \delta \bar{q}_{2}}-K_{\delta q_{1} \delta \bar{q}_{1}} K_{\delta q_{2} \delta \bar{q}_{2}}^{\prime}\right)\right] \int d^{2} \boldsymbol{y} f_{\delta q_{1} \delta q_{2}} \bar{f}_{\delta \bar{q}_{1} \delta \bar{q}_{2}}\right\} \\
& \quad+\{\text { flavor interference }\}+\{q \bar{q} \text { permutations }\} .
\end{aligned}
$$

According to (2.11) the distribution $f_{\delta q_{1} \delta q_{2}}$ describes the correlation between the directions of the transverse polarizations of two quarks in the proton. This correlation and its counterpart for antiquarks induce a correlation between the leptonic decay planes of the vector bosons, even if their transverse momenta are integrated over. Only if one integrates over the azimuthal angle of at least one of the leptons does the contribution from transverse quark polarization completely disappear from the cross section.

The cross section of the double Drell-Yan process with two photons was calculated in [46], integrated over the transverse boson momenta and over the angles of the decay leptons. The expression in equation (9) of [46] agrees with our result (3.6) (up to the combinatorial factor $1 / C$, which was omitted in [46]).

\section{Transverse position dependence of distributions}

So far we have focused on spin correlations in DPDs and their consequences for the double Drell-Yan cross section. Even for unpolarized partons, however, there can be correlations between two partons in the proton, namely correlations affecting the dependence of DPDs on the transverse variables $\boldsymbol{y}, \boldsymbol{z}_{1}$ and $\boldsymbol{z}_{2}$ and the interplay between these variables and the longitudinal momentum fractions.

In the present section we take a brief look at this issue by using a simple model in which the proton is described by a three-quark wave function. This is clearly too simple to describe the physics of small momentum fractions most relevant at the LHC, although it may actually be used for modeling quark DPDs at momentum fractions in the valence region. We proceed with this model in the spirit of an exploratory study.

Our model ansatz for the three-quark light-cone wave function of the proton is

$$
\Psi\left(x_{i}, \boldsymbol{b}_{i}-\boldsymbol{b}\right)=\Phi\left(x_{i}\right) \exp \left[-\frac{1}{4 a^{2}} \sum_{i=1}^{3} x_{i}\left(\boldsymbol{b}_{i}-\boldsymbol{b}\right)^{2}\right],
$$


where $a$ is parameter of dimension length, $\boldsymbol{b}=x_{1} \boldsymbol{b}_{1}+x_{2} \boldsymbol{b}_{2}+x_{3} \boldsymbol{b}_{3}$ is the transverse position of the proton, and $x_{1}+x_{2}+x_{3}=1$. The corresponding wave function depending on transverse momenta is a Gaussian with exponent $-a^{2} \sum_{i} \boldsymbol{k}_{i}^{2} / x_{i}$, which was long ago proposed in [65] and is often used for the phenomenology of valence dominated quantities, see e.g. [66]. The relation between the light-cone wave functions in transverse momentum and transverse position representation can be found in [67]. We do not specify the longitudinal part $\Phi\left(x_{i}\right)$ of the wave function nor its spin-flavor dependence here, since the focus of our study is on the transverse variables.

From the light-cone wave function (4.1) one can compute the contribution of the threequark Fock state to the DPD of two quarks in the proton, in full analogy to the well-known case of single-parton distributions (discussed e.g. in [67]). Up to a factor depending only on the longitudinal momentum fractions $x_{i}$, the DPD is given by

$$
\begin{aligned}
F\left(x_{i}, \boldsymbol{z}_{i}, \boldsymbol{y}\right) \propto & \exp \left[-\frac{1}{8 a^{2}}\left\{x_{1}\left(1-x_{1}\right) \boldsymbol{z}_{1}^{2}-2 x_{1} x_{2} \boldsymbol{z}_{1} \boldsymbol{z}_{2}+x_{2}\left(1-x_{2}\right) \boldsymbol{z}_{2}^{2}+\frac{4 x_{1} x_{2}}{x_{1}+x_{2}} \boldsymbol{y}^{2}\right\}\right] \\
& \times \int d^{2} \boldsymbol{b} \exp \left[-\frac{1}{2 a^{2}} \frac{x_{1}+x_{2}}{1-x_{1}-x_{2}}\left(\boldsymbol{b}+\frac{x_{1}}{x_{1}+x_{2}} \boldsymbol{y}\right)^{2}\right]
\end{aligned}
$$

where $\boldsymbol{b}$ is the transverse position of the proton, averaged over the scattering amplitude and its conjugate as specified in [40]. The second line in (4.2) just gives an $x_{i}$ dependent factor after integration over $\boldsymbol{b}$.

Inserting (4.2) into the cross section formula (2.1) and performing the integrals over all transverse positions, one obtains a cross section for double hard scattering that depends on the transverse boson momenta as

$$
\exp \left\{-a^{2}\left[\boldsymbol{q}_{1}^{2} C_{11}\left(x_{i}, \bar{x}_{i}\right)+2 \boldsymbol{q}_{1} \boldsymbol{q}_{2} C_{12}\left(x_{i}, \bar{x}_{i}\right)+\boldsymbol{q}_{2}^{2} C_{22}\left(x_{i}, \bar{x}_{i}\right)\right]\right\}
$$

with dimensionless functions $C_{i j}$ of the momentum fractions $x_{1}, x_{2}$ and $\bar{x}_{1}, \bar{x}_{2}$, which are somewhat lengthy and will not be given here. The expression in square brackets is positive definite, so that the transverse momentum dependence has a Gaussian falloff at large transverse momenta. The coefficient $C_{12}$ describing the correlation between $\boldsymbol{q}_{1}$ and $\boldsymbol{q}_{2}$ is positive as well, so that one finds a preference for the two vector bosons to have opposite transverse momenta. We see that even with the simple wave function ansatz (4.1) the dependence of the cross section on the transverse momenta of the gauge bosons is not independent of their longitudinal momenta.

An ansatz often made in phenomenology is to neglect correlations between partons and to write a collinear DPD as the convolution of two single-parton distributions that depend on the momentum fraction and the transverse position of the parton. This ansatz can be extended to include $\boldsymbol{z}_{i}$ dependent DPDs and then reads

$$
F\left(x_{i}, \boldsymbol{z}_{i}, \boldsymbol{y}\right) \approx \int d^{2} \boldsymbol{b} f\left(x_{2}, \boldsymbol{z}_{2} ; \boldsymbol{b}+\frac{1}{2} x_{1} \boldsymbol{z}_{1}\right) f\left(x_{1}, \boldsymbol{z}_{1} ; \boldsymbol{b}+\boldsymbol{y}-\frac{1}{2} x_{2} \boldsymbol{z}_{2}\right) .
$$

The second argument of the single-parton distribution $f$ is Fourier conjugate to the transverse quark momentum and the third argument gives the transverse position of the proton 
with respect to the quark, both averaged over the scattering amplitude and its conjugate. The shift of this argument by $\frac{1}{2} x_{1} z_{1}$ or $-\frac{1}{2} x_{2} z_{2}$ is a consequence of Lorentz invariance as explained in [39]. Evaluating the single-parton distributions for the light-cone wave function (4.1) one obtains

$$
\begin{aligned}
& F\left(x_{i}, \boldsymbol{z}_{i}, \boldsymbol{y}\right) \propto \int d^{2} \boldsymbol{b} \exp {\left[-\frac{1}{8 a^{2}} \frac{x_{2}}{1-x_{2}}\left\{\left(1-x_{2}\right)^{2} \boldsymbol{z}_{2}^{2}+\left(2 \boldsymbol{b}+x_{1} \boldsymbol{z}_{1}\right)^{2}\right\}\right] } \\
& \times \exp \left[-\frac{1}{8 a^{2}} \frac{x_{1}}{1-x_{1}}\left\{\left(1-x_{1}\right)^{2} \boldsymbol{z}_{1}^{2}+\left(2 \boldsymbol{b}+2 \boldsymbol{y}-x_{2} \boldsymbol{z}_{2}\right)^{2}\right\}\right] \\
& \propto \exp \left[-\frac{1}{8 a^{2}}\left\{x_{1}\left(1-x_{1}\right) \boldsymbol{z}_{1}^{2}+x_{2}\left(1-x_{2}\right) \boldsymbol{z}_{2}^{2}\right.\right. \\
&\left.\left.+\frac{x_{1} x_{2}}{x_{1}\left(1-x_{2}\right)+\left(1-x_{1}\right) x_{2}}\left(2 \boldsymbol{y}-x_{1} \boldsymbol{z}_{1}-x_{2} \boldsymbol{z}_{2}\right)^{2}\right\}\right]
\end{aligned}
$$

for the transverse dependence of the DPD. This is visibly different from the result (4.2) of the direct calculation. Although the ansatz (4.4) involves the convolution of two singleparton distributions, thus suggesting that the two partons are distributed independently, it induces correlations between transverse and longitudinal variables in $F\left(x_{i}, \boldsymbol{z}_{i}, \boldsymbol{y}\right)$.

Inserting the form (4.5) into the cross section formula one obtains again a Gaussian behavior as in (4.3), but with different coefficients $C_{i j}$. In particular, the sign of $C_{12}$ is then equal to the sign of $\left(x_{1}-\bar{x}_{1}\right)\left(x_{2}-\bar{x}_{2}\right)$, so that depending on the longitudinal momentum fractions the transverse boson momenta tend to be in the same hemisphere or in opposite ones. This difference in qualitative behavior shows that the ansatz (4.4) must be used with great care when one is interested in correlation effects.

Setting $\boldsymbol{z}_{1}=\boldsymbol{z}_{2}=\mathbf{0}$ in (4.2) and (4.5) gives collinear DPDs with a Gaussian dependence on $\boldsymbol{y}$. The Gaussian width depends on $x_{1}$ and $x_{2}$ and differs in the two cases,

$$
\begin{aligned}
& \left.F\left(x_{i}, \boldsymbol{y}\right)\right|_{(4.2)} \propto \exp \left[-\frac{1}{2 a^{2}} \frac{x_{1} x_{2}}{x_{1}+x_{2}} \boldsymbol{y}^{2}\right], \\
& \left.F\left(x_{i}, \boldsymbol{y}\right)\right|_{(4.5)} \propto \exp \left[-\frac{1}{2 a^{2}} \frac{x_{1} x_{2}}{x_{1}+x_{2}-2 x_{1} x_{2}} \boldsymbol{y}^{2}\right] .
\end{aligned}
$$

We see that, within our model, the ansatz (4.4) does not reproduce the interplay between $\boldsymbol{y}$ and the momentum fractions. It does, however, provide a valid approximation unless $x_{1}$ and $x_{2}$ are both rather large.

\section{Summary}

Multiple hard interactions in $p p$ collisions can yield substantial contributions to the production of final states with high multiplicity in parts of phase space. In this paper we have shown how spin correlations between two partons in the proton affect the rate and the angular distribution of the final state in the production of four leptons via two electroweak gauge bosons. We considered both the case where the transverse momenta of the bosons are small (using transverse-momentum dependent factorization) and the case where they are integrated over (using collinear factorization). 
We find that longitudinal spin correlations between the quarks or antiquarks in the proton affect the rate of double parton scattering, and in the presence of axial-vector currents also the polar distribution of the produced leptons. Correlations involving transversely polarized quarks or antiquarks induce azimuthal correlations between the final state leptons. A part of these correlations persists if the transverse momenta of the gauge bosons are integrated over. Having two "independent" hard interactions in double parton scattering does hence not imply that the final states produced by the two interactions are independent of each other.

How large parton spin correlations inside a proton actually are remains an open question that deserves further study. This also holds for possible correlations between the transverse distribution and the longitudinal momentum fractions of the partons. We find several such correlations in a simple model with a three-quark wave function. Within this model we also find that the often used ansatz to represent double parton distributions as convolutions of single-parton distributions is inadequate to describe details of the kinematic dependence in double parton scattering.

Double Drell-Yan production involves a particularly simple hard-scattering subprocess but nevertheless exhibits a rich pattern of angular effects induced by parton spin correlations. It is natural to expect that other processes, in particular those involving multijets, will share this feature. We note that the cross section dependence on angles between the final-state particles implies a dependence on the invariant mass of particle pairs, which is an important quantity in searches for new physics. An estimate of the possible size of such effects would therefore be of great value.

\section{Acknowledgments}

It is our pleasure to thank J. Gaunt and L. Zeune for valuable remarks on the manuscript.

\section{A Coupling factors}

In this appendix we list the coupling factors $K$ and $K^{\prime}$ appearing in the double Drell-Yan cross section. Further relations between these factors are given in section 2.3.

\section{A.1 Charged vector bosons}

For $W^{+}$production one has

$$
K_{q_{i} \bar{q}_{j}}=\frac{\alpha^{2}}{4 N_{c}} \frac{\left|V_{q_{i} q_{j}}\right|^{2}}{\left(2 \sin \theta_{w}\right)^{4}} \frac{Q_{i}^{2}}{\left(Q_{i}^{2}-m_{W}^{2}\right)^{2}+m_{W}^{2} \Gamma_{W}^{2}}, \quad\left(e_{q_{i}}-e_{q_{j}}=1\right)
$$

and for $W^{-}$production

$$
K_{q_{i} \bar{q}_{j}}=\frac{\alpha^{2}}{4 N_{c}} \frac{\left|V_{q_{j} q_{i}}\right|^{2}}{\left(2 \sin \theta_{w}\right)^{4}} \frac{Q_{i}^{2}}{\left(Q_{i}^{2}-m_{W}^{2}\right)^{2}+m_{W}^{2} \Gamma_{W}^{2}}, \quad\left(e_{q_{i}}-e_{q_{j}}=-1\right) .
$$

Here $N_{c}=3$ is the number of colors, $V_{q_{i} q_{j}}$ a CKM matrix element, $\theta_{w}$ the weak mixing angle, $\alpha$ the electromagnetic fine structure constant, and $e_{q_{i}}$ the charge of quark $q_{i}$ in units of the positron charge. 


\section{A.2 Neutral vector bosons}

For a lepton pair $\ell^{+} \ell^{-}$produced via a $\gamma^{*}, Z$ or their interference, one has coupling factors

$$
\begin{aligned}
& K_{q_{i} \bar{q}_{j}}=\frac{\alpha^{2}}{4 N_{c}}\left\{\frac{e_{q_{i}} e_{q_{j}}}{Q_{i}^{2}}-A\left(Q_{i}\right) g_{\ell}^{V}\left(e_{q_{i}} g_{q_{j}}^{V}+e_{q_{j}} g_{q_{i}}^{V}\right)-i B\left(Q_{i}\right) g_{\ell}^{V}\left(e_{q_{i}} g_{q_{j}}^{V}-e_{q_{j}} g_{q_{i}}^{V}\right)\right. \\
&+\left.+C\left(Q_{i}\right)\left[\left(g_{\ell}^{V}\right)^{2}+\left(g_{\ell}^{A}\right)^{2}\right]\left(g_{q_{i}}^{V} g_{q_{j}}^{V}+g_{q_{j}}^{A} g_{q_{i}}^{A}\right)\right\}, \\
& K_{q_{i} \bar{q}_{j}}^{\prime}=\frac{\alpha^{2}}{4 N_{c}}\left\{-A\left(Q_{i}\right) g_{\ell}^{A}\left(e_{q_{i}} g_{q_{j}}^{A}+e_{q_{j}} g_{q_{i}}^{A}\right)-i B\left(Q_{i}\right) g_{\ell}^{A}\left(e_{q_{i}} g_{q_{j}}^{A}-e_{q_{j}} g_{q_{i}}^{A}\right)\right. \\
&\left.+C\left(Q_{i}\right) 2 g_{\ell}^{V} g_{\ell}^{A}\left(g_{q_{i}}^{V} g_{q_{j}}^{A}+g_{q_{j}}^{V} g_{q_{i}}^{A}\right)\right\}, \\
& K_{q_{i} \Delta \bar{q}_{j}}=\frac{\alpha^{2}}{4 N_{c}}\left\{-A\left(Q_{i}\right) g_{\ell}^{V}\left(e_{q_{i}} g_{q_{j}}^{A}+e_{q_{j}} g_{q_{i}}^{A}\right)-i B\left(Q_{i}\right) g_{\ell}^{V}\left(e_{q_{i}} g_{q_{j}}^{A}-e_{q_{j}} g_{q_{i}}^{A}\right)\right. \\
&\left.+C\left(Q_{i}\right)\left[\left(g_{\ell}^{V}\right)^{2}+\left(g_{\ell}^{A}\right)^{2}\right]\left(g_{q_{i}}^{V} g_{q_{j}}^{A}+g_{q_{j}}^{V} g_{q_{i}}^{A}\right)\right\}, \\
& K_{q_{i} \Delta \bar{q}_{j}}^{\prime}=\frac{\alpha^{2}}{4 N_{c}}\left\{-A\left(Q_{i}\right) g_{\ell}^{A}\left(e_{q_{i}} g_{q_{j}}^{V}+e_{q_{j}} g_{q_{i}}^{V}\right)-i B\left(Q_{i}\right) g_{\ell}^{A}\left(e_{q_{i}} g_{q_{j}}^{V}-e_{q_{j}} g_{q_{i}}^{V}\right)\right. \\
&\left.+C\left(Q_{i}\right) 2 g_{\ell}^{V} g_{\ell}^{A}\left(g_{q_{i}}^{V} g_{q_{j}}^{V}+g_{q_{j}}^{A} g_{q_{i}}^{A}\right)\right\}
\end{aligned}
$$

and

$$
\begin{aligned}
K_{\delta q_{i} \delta \bar{q}_{j}}=\frac{\alpha^{2}}{4 N_{c}} & \left\{\frac{e_{q_{i}} e_{q_{j}}}{Q_{i}^{2}}-A\left(Q_{i}\right) g_{\ell}^{V}\left(e_{q_{i}} g_{q_{j}}^{V}+e_{q_{j}} g_{q_{i}}^{V}\right)-i B\left(Q_{i}\right) g_{\ell}^{V}\left(e_{q_{i}} g_{q_{j}}^{V}-e_{q_{j}} g_{q_{i}}^{V}\right)\right. \\
+ & \left.C\left(Q_{i}\right)\left[\left(g_{\ell}^{V}\right)^{2}+\left(g_{\ell}^{A}\right)^{2}\right]\left(g_{q_{i}}^{V} g_{q_{j}}^{V}-g_{q_{j}}^{A} g_{q_{i}}^{A}\right)\right\}, \\
K_{\delta q_{i} \delta \bar{q}_{j}}^{\prime}=\frac{\alpha^{2}}{4 N_{c}}\{ & -B\left(Q_{i}\right) g_{\ell}^{V}\left(e_{q_{i}} g_{q_{j}}^{A}+e_{q_{j}} g_{q_{i}}^{A}\right)+i A\left(Q_{i}\right) g_{\ell}^{V}\left(e_{q_{i}} g_{q_{j}}^{A}-e_{q_{j}} g_{q_{i}}^{A}\right) \\
& \left.-i C\left(Q_{i}\right)\left[\left(g_{\ell}^{V}\right)^{2}+\left(g_{\ell}^{A}\right)^{2}\right]\left(g_{q_{i}}^{V} g_{q_{j}}^{A}-g_{q_{j}}^{V} g_{q_{i}}^{A}\right)\right\} .
\end{aligned}
$$

Here we have used the conventional vector and axial fermion couplings to the $Z$ boson,

$$
g_{f}^{V}=I_{f}^{3}-2 e_{f} \sin ^{2} \theta_{w}, \quad g_{f}^{A}=I_{f}^{3},
$$

where $I_{f}^{3}$ is the third component of the weak isospin of the left handed fermion $f$ and $e_{f}$ its charge in units of positron charge. Since we do not consider $Z$ decays to neutrinos, $\ell$ is always a negatively charged lepton. We have furthermore used the abbreviations

$$
\begin{aligned}
& A\left(Q_{i}\right)=\frac{1}{\sin ^{2} 2 \theta_{w}} \frac{Q_{i}^{2}-m_{Z}^{2}}{\left(Q_{i}^{2}-m_{Z}^{2}\right)^{2}+m_{Z}^{2} \Gamma_{Z}^{2}}, \quad B\left(Q_{i}\right)=\frac{1}{\sin ^{2} 2 \theta_{w}} \frac{m_{Z} \Gamma_{Z}}{\left(Q_{i}^{2}-m_{Z}^{2}\right)^{2}+m_{Z}^{2} \Gamma_{Z}^{2}}, \\
& C\left(Q_{i}\right)=\frac{1}{\sin ^{4} 2 \theta_{w}} \frac{Q_{i}^{2}}{\left(Q_{i}^{2}-m_{Z}^{2}\right)^{2}+m_{Z}^{2} \Gamma_{Z}^{2}} .
\end{aligned}
$$

For the usual hard-scattering cross sections one has equal flavors $q_{i}=q_{j}$ in the above coupling factors and finds that their imaginary parts are zero. This is not the case for the coupling factors describing flavor interference, where $q_{i} \neq q_{j}$. 
Open Access. This article is distributed under the terms of the Creative Commons Attribution License which permits any use, distribution and reproduction in any medium, provided the original author(s) and source are credited.

\section{References}

[1] A. Del Fabbro and D. Treleani, A double parton scattering background to Higgs boson production at the LHC, Phys. Rev. D 61 (2000) 077502 [hep-ph/9911358] [INSPIRE].

[2] R. Godbole, S. Gupta and J. Lindfors, Double parton scattering contribution to $W+$ jets, Z. Phys. C 47 (1990) 69 [inSPIRE].

[3] O.J. Eboli, F. Halzen and J. Mizukoshi, The associated production of weak bosons and jets by multiple parton interactions, Phys. Rev. D 57 (1998) 1730 [hep-ph/9710443] [INSPIRE].

[4] E. Cattaruzza, A. Del Fabbro and D. Treleani, Fractional momentum correlations in multiple production of $W$ bosons and of $b \bar{b}$ pairs in high energy pp collisions, Phys. Rev. D 72 (2005) 034022 [hep-ph/0507052] [INSPIRE].

[5] E. Maina, Multiple parton interactions, top-antitop and $W+4 j$ production at the LHC, JHEP 04 (2009) 098 [arXiv:0904.2682] [INSPIRE].

[6] E. Maina, Multiple parton interactions in $Z+4 j W^{+-} W^{+-}+0 / 2 j$ and $W^{+} W^{-}+2 j$ production at the LHC, JHEP 09 (2009) 081 [arXiv:0909.1586] [INSPIRE].

[7] E. Maina, Multiple parton interactions in $Z^{+}$jets production at the LHC. A comparison of factorized and non-factorized double parton distribution functions, JHEP 01 (2011) 061 [arXiv: 1010.5674] [INSPIRE].

[8] A. Kulesza and W.J. Stirling, Like sign W boson production at the LHC as a probe of double parton scattering, Phys. Lett. B 475 (2000) 168 [hep-ph/9912232] [INSPIRE].

[9] J.R. Gaunt, C.-H. Kom, A. Kulesza and W.J. Stirling, Same-sign W pair production as a probe of double parton scattering at the LHC, Eur. Phys. J. C 69 (2010) 53 [arXiv: 1003.3953] [INSPIRE].

[10] E.L. Berger, C. Jackson, S. Quackenbush and G. Shaughnessy, Calculation of $W B \bar{b}$ production via double parton scattering at the LHC, Phys. Rev. D 84 (2011) 074021 [arXiv:1107.3150] [INSPIRE].

[11] B. Humpert, Are there multi-quark interactions?, Phys. Lett. B 131 (1983) 461 [InSPIRE].

[12] B. Humpert and R. Odorico, Multiparton scattering and QCD radiation as sources of four jet events, Phys. Lett. B 154 (1985) 211 [inSPIRE].

[13] L. Ametller, N. Paver and D. Treleani, Possible signature of multiple parton interactions in collider four jet events, Phys. Lett. B 169 (1986) 289 [INSPIRE].

[14] M.L. Mangano, Four jet production at the Tevatron collider, Z. Phys. C 42 (1989) 331 [INSPIRE].

[15] M. Drees and T. Han, Signals for double parton scattering at the Fermilab Tevatron, Phys. Rev. Lett. 77 (1996) 4142 [hep-ph/9605430] [INSPIRE].

[16] A. Del Fabbro and D. Treleani, Double parton scatterings in B quark pairs production at the CERN LHC, Phys. Rev. D 66 (2002) 074012 [hep-ph/0207311] [INSPIRE]. 
[17] S. Domdey, H.-J. Pirner and U.A. Wiedemann, Testing the scale dependence of the scale factor $\delta_{\mathrm{eff}}$ in double dijet production at the LHC, Eur. Phys. J. C 65 (2010) 153 [arXiv: 0906.4335] [INSPIRE].

[18] E.L. Berger, C. Jackson and G. Shaughnessy, Characteristics and estimates of double parton scattering at the large hadron collider, Phys. Rev. D 81 (2010) 014014 [arXiv:0911.5348] [INSPIRE].

[19] Axial Field Spectrometer collaboration, T. Akesson et al., Double parton scattering in pp collisions at $\sqrt{s}=63 \mathrm{GeV}, \mathrm{Z}$. Phys. C 34 (1987) 163 [InSPIRE].

[20] UA2 collaboration, J. Alitti et al., A study of multi-jet events at the CERN $\bar{p} p$ collider and a search for double parton scattering, Phys. Lett. B 268 (1991) 145 [InSPIRE].

[21] CDF collaboration, F. Abe et al., Study of four jet events and evidence for double parton interactions in pp collisions at $\sqrt{s}=1.8$ TeV, Phys. Rev. D 47 (1993) 4857 [INSPIRE].

[22] CDF collaboration, F. Abe et al., Measurement of double parton scattering in $\bar{p} p$ collisions at $\sqrt{s}=1.8$ TeV, Phys. Rev. Lett. 79 (1997) 584 [INSPIRE].

[23] CDF collaboration, F. Abe et al., Double parton scattering in $\bar{p} p$ collisions at $\sqrt{s}=1.8 \mathrm{TeV}$, Phys. Rev. D 56 (1997) 3811 [INSPIRE].

[24] D0 collaboration, V. Abazov et al., Double parton interactions in photon +3 jet events in $p \bar{p}$ collisions $\sqrt{s}=1.96 \mathrm{TeV}$, Phys. Rev. D 81 (2010) 052012 [arXiv:0912.5104] [INSPIRE].

[25] D0 collaboration, V.M. Abazov et al., Azimuthal decorrelations and multiple parton interactions in photon+2 jet and photon+3 jet events in p p collisions at $\sqrt{s}=1.96 \mathrm{TeV}$, Phys. Rev. D 83 (2011) 052008 [arXiv:1101.1509] [INSPIRE].

[26] ATLAS collaboration, A measurement of hard double-partonic interactions in $W \rightarrow l \nu+2$ jet events using the ATLAS detector at the LHC, ATLAS-CONF-2011-160 (2011).

[27] CMS collaboration, P. Bartalini and L. Fano, Multiple parton interactions studies at CMS, arXiv:1103.6201 [INSPIRE].

[28] T. Sjöstrand and M. van Zijl, Multiple parton-parton interactions in an impact parameter picture, Phys. Lett. B 188 (1987) 149 [INSPIRE].

[29] T. Sjöstrand and M. van Zijl, A multiple interaction model for the event structure in hadron collisions, Phys. Rev. D 36 (1987) 2019 [InSPIRE].

[30] J. Butterworth, J.R. Forshaw and M. Seymour, Multiparton interactions in photoproduction at HERA, Z. Phys. C 72 (1996) 637 [hep-ph/9601371] [InSPIRE].

[31] T. Sjöstrand and P.Z. Skands, Multiple interactions and the structure of beam remnants, JHEP 03 (2004) 053 [hep-ph/0402078] [INSPIRE].

[32] M. Bahr, S. Gieseke and M.H. Seymour, Simulation of multiple partonic interactions in HERWIG++, JHEP 07 (2008) 076 [arXiv:0803.3633] [INSPIRE].

[33] R. Corke and T. Sjöstrand, Multiparton interactions and rescattering, JHEP 01 (2010) 035 [arXiv: 0911.1909] [INSPIRE].

[34] R. Corke and T. Sjöstrand, Multiparton interactions with an x-dependent proton size, JHEP 05 (2011) 009 [arXiv: 1101.5953] [INSPIRE].

[35] e. Bartalini, Paolo and e. Fano, Livio, Multiple partonic interactions at the LHC. Proceedings, 1st international workshop, MPI'08, Perugia, Italy, October 27-31, 2008, arXiv: 1003.4220 [INSPIRE]. 
[36] P. Bartalini, E. Berger, B. Blok, G. Calucci, R. Corke, et al., Multi-parton interactions at the $L H C$, arXiv: 1111.0469 [INSPIRE].

[37] B. Blok, Y. Dokshitzer, L. Frankfurt and M. Strikman, The four jet production at LHC and Tevatron in QCD, Phys. Rev. D 83 (2011) 071501 [arXiv: 1009.2714] [InSPIRE].

[38] B. Blok, Y. Dokshitser, L. Frankfurt and M. Strikman, $p Q C D$ physics of multiparton interactions, Eur. Phys. J. C 72 (2012) 1963 [arXiv:1106.5533] [INSPIRE].

[39] M. Diehl and A. Schafer, Theoretical considerations on multiparton interactions in QCD, Phys. Lett. B 698 (2011) 389 [arXiv:1102.3081] [INSPIRE].

[40] M. Diehl, D. Ostermeier and A. Schafer, Elements of a theory for multiparton interactions in QCD, JHEP 03 (2012) 089 [arXiv:1111.0910] [INSPIRE].

[41] J. Bartels and M. Ryskin, Recombination within multi-chain contributions in pp scattering, arXiv:1105.1638 [INSPIRE].

[42] M. Ryskin and A. Snigirev, A fresh look at double parton scattering, Phys. Rev. D 83 (2011) 114047 [arXiv:1103.3495] [INSPIRE].

[43] M. Ryskin and A. Snigirev, Double parton scattering in double logarithm approximation of perturbative QCD, Phys. Rev. D 86 (2012) 014018 [arXiv:1203.2330] [INSPIRE].

[44] J.R. Gaunt and W.J. Stirling, Double parton scattering singularity in one-loop integrals, JHEP 06 (2011) 048 [arXiv: 1103.1888] [INSPIRE].

[45] J.R. Gaunt, Single perturbative splitting diagrams in double parton scattering, arXiv: 1207.0480 [INSPIRE].

[46] A.V. Manohar and W.J. Waalewijn, A QCD analysis of double parton scattering: color correlations, interference effects and evolution, Phys. Rev. D 85 (2012) 114009 [arXiv: 1202.3794] [INSPIRE].

[47] A.V. Manohar and W.J. Waalewijn, What is double parton scattering?, Phys. Lett. B 713 (2012) 196 [arXiv:1202.5034] [INSPIRE].

[48] G. Calucci and D. Treleani, Mini-jets and the two-body parton correlation, Phys. Rev. D 57 (1998) 503 [hep-ph/9707389] [INSPIRE].

[49] G. Calucci and D. Treleani, Proton structure in transverse space and the effective cross-section, Phys. Rev. D 60 (1999) 054023 [hep-ph/9902479] [INSPIRE].

[50] A. Del Fabbro and D. Treleani, Scale factor in double parton collisions and parton densities in transverse space, Phys. Rev. D 63 (2001) 057901 [hep-ph/0005273] [INSPIRE].

[51] T. Rogers and M. Strikman, Multiple hard partonic collisions with correlations in proton-proton scattering, Phys. Rev. D 81 (2010) 016013 [arXiv:0908.0251] [INSPIRE].

[52] C. Flensburg, G. Gustafson, L. Lönnblad and A. Ster, Correlations in double parton distributions at small $x$, JHEP 06 (2011) 066 [arXiv:1103.4320] [INSPIRE].

[53] M. Mekhfi, Correlations in color and spin in multiparton processes, Phys. Rev. D 32 (1985) 2380 [INSPIRE].

[54] C. Goebel, F. Halzen and D. Scott, Double Drell-Yan annihilations in hadron collisions: novel tests of the constituent picture, Phys. Rev. D 22 (1980) 2789 [InSPIRE].

[55] M. Mekhfi, Multiparton processes: an application to double Drell-Yan, Phys. Rev. D 32 (1985) 2371 [inSPIRE]. 
[56] F. Halzen, P. Hoyer and W.J. Stirling, Evidence for multiple parton interactions from the observation of multi-muon events in Drell-Yan experiments, Phys. Lett. B 188 (1987) 375 [INSPIRE].

[57] C. Kom, A. Kulesza and W. Stirling, Prospects for observation of double parton scattering with four-muon final states at LHCb, Eur. Phys. J. C 71 (2011) 1802 [arXiv:1109.0309] [INSPIRE].

[58] J.C. Collins and D.E. Soper, Back-to-back jets in QCD, Nucl. Phys. B 193 (1981) 381 [Erratum ibid. B 213 (1983) 545] [INSPIRE].

[59] J.C. Collins, The foundations of perturbative QCD, Cambridge University Press, Cambridge U.K. (2011).

[60] N. Paver and D. Treleani, Multi-quark scattering and large $p_{T}$ jet production in hadronic collisions, Nuovo Cim. A 70 (1982) 215 [INSPIRE].

[61] J.C. Collins and D.E. Soper, Angular distribution of dileptons in high-energy hadron collisions, Phys. Rev. D 16 (1977) 2219 [InSPIRE].

[62] D. Boer and W. Vogelsang, Drell-Yan lepton angular distribution at small transverse momentum, Phys. Rev. D 74 (2006) 014004 [hep-ph/0604177] [INSPIRE].

[63] S. Arnold, A. Metz and M. Schlegel, Dilepton production from polarized hadron hadron collisions, Phys. Rev. D 79 (2009) 034005 [arXiv: 0809.2262] [INSPIRE].

[64] D. Boer, Investigating the origins of transverse spin asymmetries at RHIC, Phys. Rev. D 60 (1999) 014012 [hep-ph/9902255] [INSPIRE].

[65] S. J. Brodsky, T. Huang and G. P. Lepage, The hadronic wave function in quantum chromodynamics, SLAC-PUB-2540 (2001).

[66] M. Diehl, T. Feldmann, R. Jakob and P. Kroll, Linking parton distributions to form factors and Compton scattering, Eur. Phys. J. C 8 (1999) 409 [hep-ph/9811253].

[67] M. Diehl, Generalized parton distributions, Phys. Rept. 388 (2003) 41 [hep-ph/0307382] [INSPIRE]. 